\title{
A Novel Nanobody-Heavy Chain Antibody Against Angiopoietin-Like Protein 3 Reduces Plasma Lipids and Relieves Nonalcoholic Fatty Liver Disease
}

Xiaozhi Hu

Fudan University

Jiajun Fan

Fudan University

Qianqian Ma

Fudan University

Lei Han

Fudan University

Zhonglian Cao

Fudan University

Caili Xu

Fudan University

Jingyun Luan

University of Chicago Division of the Biological Sciences

Yanyang Nan

Fudan University

Tao Wu

Fudan University

Yuting Zhang

Fudan University

Hanqi Wang

Fudan University

Dianwen Ju ( $\nabla$ dianwenju@fudan.edu.cn )

Fudan University

\section{Research Article}

Keywords: ANGPTL3, nanobody, lipid-reducing, hypercholesterolemia, NAFLD

Posted Date: January 21st, 2022

DOI: https://doi.org/10.21203/rs.3.rs-1242140/v1 
License: (c) (i) This work is licensed under a Creative Commons Attribution 4.0 International License. Read Full License 


\section{Abstract \\ Background}

Nonalcoholic fatty liver disease (NAFLD) is a metabolic disease mainly on account of

hypercholesterolemia and may progresses to cirrhosis and hepatocellular carcinoma. The discovery of effective therapies for NAFLD is a critical unmet need. Angiopoietin-like protein 3 (ANGPTL3), a critical lipid metabolism regulator, cause elevated blood lipids and are elevated in NAFLD. Here, we develop a nanobody-heavy chain antibody (VHH-Fc) to inhibit ANGPTL3 for NAFLD treatment.

\section{Results}

In this study, we screened out a anti-ANGPTL3 VHH and Fc fusion protein, C44-Fc, which exhibited a high affinity to human and mouse ANGPTL3 proteins at 0.1402 0.2088 nM and rescued ANGPLT3-mediated inhibition of lipoprotein lipase (LPL) activity. The C44-Fc had different ANGPTL3 binding epitope, a double expression yield and better thermostability when compared with the evinacumab, maintaining no obvious degradation or aggregation at low and high $\mathrm{pH}, 40^{\circ} \mathrm{C}$ for 28 days, and freeze-thaw. Furthermore, C44-Fc significantly ameliorated the triglyceride ( 44.2\%), total cholesterol ( 36.6\%) and LDL-cholesterol $(\sim 54.4 \%)$ levels in hypercholesterolemic mice and ameliorated hepatic lipid accumulation and liver injury in NAFLD mice.

\section{Conclusions}

We discover a VHH-Fc fusion protein with high affinity to ANGPTL3 and alleviates the progression of NAFLD, which offers a prospective therapy for NAFLD.

\section{Background}

Hypercholesterolemia, accompanied by increased total cholesterol (TC) including low-density lipoprotein (LDL), shows positive correlation with nonalcoholic fatty liver disease (NAFLD). ${ }^{1,2}$ As one of the major metabolic organs, the liver is closely regulated in response to hypercholesterolemia, undergoes steatosis and then evolves into NAFLD. ${ }^{3}$ NAFLD is the most prevalent liver disease and characterized by abnormal hepatic triglyceride $(\mathrm{TG})$ and cholesterol accumulations. Its pathologies range from nonalcoholic steatohepatitis to cirrhosis and even progressed to hepatocellular carcinoma. ${ }^{4}$ Although there have been advances in lipid-lowering therapy including peroxisome proliferation activating receptors (PPAR)- $\mathrm{a} / \mathrm{Y}$ agonists and statins, NAFLD still afflict 1.7 billion population worldwide. ${ }^{5-7}$ Therefore, novel treatment for NAFLD is imminently required.

Angiopoietin-like protein 3 (ANGPTL3), as a lipometabolic regulator, was a secretory protein and primarily expressed in the liver. ${ }^{8}$ It could be cleaved into a coiled-coil domain (CCD) and a fibrinogen-like domain 
(FLD). The CCD and the full-length of ANGPTL3 all could inhibit the enzyme activity of lipoprotein lipase $(\mathrm{LPL})$ as well as endothelial lipase (EL). ${ }^{8-11}$ The individuals of ANGPTL3-function deficiency exist inartificially with low plasma TG and TC levels and display no clinical risks. ${ }^{12,13}$ It has been well documented that the high-cholesterol diet can carry out elevated ANGPTL3 expression levels as a consequence of liver $X$ receptors (LXRs) activated, which aggravated hypertriglyceridemia. ${ }^{14,15}$ Besides, ANGPTL3 had increased expression levels in the liver of NAFLD patients, which resulted in elevated circulating ANGPTL3, indicating ANGPTL3 may be a critical target in NAFLD. ${ }^{16}$ The ANGPTL3 inhibitors could efficiently ameliorate plasma TG and TC levels in hypercholesterolemic patients and relieve the progress of atherosclerosis. ${ }^{17,18}$ Nevertheless, its effect in NAFLD has not been reported.

Up to now, three diverse blockade tactics against ANGPTL3 have been validated, including antisense oligonucleotide (ASO, IONIS-ANGPTL3-LRx), the CRISPR/Cas9 gene knockout technology and the monoclonal antibody. ${ }^{19-21}$ Unfortunately, some of them have the severe limitations and their druggability remains controversial. As was reported previously, the ASO-based drugs are easily degradable and the CRISPR/Cas9 gene knockout technology have the off-target effects and ethical limitations. ${ }^{22,23}$ In addition to traditional antibody, nanobody is a camelids single domain antibody (VHH) and has nanoscale size of $\sim 15 \mathrm{kDa}$, which has various advantages over the traditional immunoglobulin gamma (IgG). ${ }^{24-26}$ It could be expressed with high yield and stably in mammalian cell, yeast and even bacteria. ${ }^{27}$, 28 It has splendid penetration even through the blood-brain barrier due to nanometer scale, superior thermostability and chemostability, preeminent specificity and binding-ability to antigen. ${ }^{28-34}$ Lately, the first nanobody drug, a bivalent VHHs fusion protein (Caplacizumab) was approved to treat thrombotic thrombocytopenic purpura by the European Commission. ${ }^{35}$ Thus, bivalent VHHs fusion protein may be a promising ANGPTL3 inhibitor as therapeutic treatment options.

In this manuscript, we aim to discover the therapeutic effect of VHHs fusion protein against ANGPTL3 on NAFLD. After immunizing an alpaca with hANGPTL3 (S17-K170), we screened out the VHH binding to ANGPTL3-CCD with high affinity, which were then fused to human IgG1-Fc to establish the fusion protein. Our results indicated that the VHH-Fc fusion protein could block ANGPTL3-mediated suppression of LPL activity in vitro, reduce serum lipid level and ameliorated hepatic lipid accumulation and liver injury in NAFLD mice.

\section{Results}

\section{Construction of the VHHs library and phage display}

After immunizing an alpaca for three times with hANGPTL3 (S17-K170), the serological ELISA was performed with $200 \mathrm{ng}$ hANGPTL3 (S17-K170) antigen/well. As shown in Additional file 1:Figure S1A, the titer of serological antibody specific to the hANGPTL3 (S17-K170) was 1:64000. The naïve phage display VHHs library was developed subsequently and its size was detected and calculated to be approximate $5 \times 10^{9}$ by counting individual transformants of TG1 E. coli with tenfold dilution. After three rounds of bio- 
panning, the specific nanobodies against hANGPTL3 (S17-K170) were enriched, and 33 clones were found to specifically bind with ANGPTL3 (Fig. 2A). Thus, all the sequences of 33 positive clones were sequenced and analyzed by multiple amino acid sequence alignment as well as the phylogenetic tree analysis. Our results showed that 11 unique nanobodies had different sequences in CDRs (Additional file 1: Fig. S2), indicating that the VHHs library had good diversity.

\section{Expression of VHHs-Fc and kinetics screening}

To improve the short half-life period of the $\mathrm{VHH}$ binder, the $11 \mathrm{VHHs}$ were fused to human IgG1 Fc domain (VHHs-Fc) to construct the alpaca-human chimeric antibody (Fig. 2B). The VHHs-Fc chimeric antibodies were then effectively expressed in ExpiCHO cells using a commercial pTT5 vector. SDS-PAGE results in Figure $2 \mathrm{C}$ demonstrated that the VHHs-Fc fusion protein was appropriately produced with high purity (78 $\mathrm{kDa}$ in non-reduced PAGE and $39 \mathrm{kDa}$ in reduced PAGE).

The $11 \mathrm{VHH-Fc}$ fusions protein candidates were then tested for the binding ability to hANGPTL3 (S17K170) by SPR. As shown in Table 1, there were four VHH- Fc fusions proteins (C27-Fc, C42-Fc, C44-Fc and $\mathrm{C} 45-\mathrm{Fc}$ ) that had superior stability and binding ability to hANGPTL3 (S17-K170) ( $\mathrm{kd}<1 \times 10^{-4}$ and Rmax > $60 \mathrm{RU})$. C27-Fc, in particular, had the highest binding ( $R \max =112.6 \mathrm{RU})$ and stability $\left(\mathrm{kd}=6.56 \times 10^{-5}\right)$ values. C42-Fc and C44-Fc binding values were approximately $110 \mathrm{RU}$, which were slightly lower than C27Fc. C44-Fc binding values were 65.1 RU, which were comparable to evinacumab (66.4RU). Altogether, we were able to identify four candidates with high and stable binding to hANGPTL3 (S17-K170). 
Table 1

The kinetic screening of the VHHs-Fc against human ANGPTL3

\begin{tabular}{|c|c|c|c|c|c|}
\hline Sample & ka (1/Ms) & $k d(1 / s)$ & $\mathrm{KD}(\mathrm{M})$ & $\operatorname{Rmax}(\mathrm{RU})$ & $\mathrm{Chi}^{2}\left(\mathrm{RU}^{2}\right)$ \\
\hline $\mathrm{C} 27-\mathrm{Fc}$ & $2.17 \mathrm{E}+06$ & 1.03E-04 & $4.72 \mathrm{E}-11$ & 112.6 & 1.47 \\
\hline $\mathrm{C} 42-\mathrm{Fc}$ & $1.81 e+06$ & $6.56 \mathrm{e}-05$ & $3.62 \mathrm{E}-11$ & 130.2 & 1.64 \\
\hline $\mathrm{C} 45-\mathrm{Fc}$ & $1.66 \mathrm{E}+06$ & $9.30 \mathrm{E}-05$ & $5.62 \mathrm{E}-11$ & 118.5 & 1.36 \\
\hline Evinacumab & $6.95 \mathrm{E}+05$ & $1.65 \mathrm{E}-04$ & 2.38E-10 & 66.4 & 0.0913 \\
\hline $\mathrm{C} 44-\mathrm{Fc}$ & $6.94 \mathrm{E}+05$ & $3.51 \mathrm{E}-04$ & 5.05E-10 & 65.1 & 0.342 \\
\hline $\mathrm{C} 26-\mathrm{Fc}$ & $1.91 \mathrm{E}+10$ & $1.91 \mathrm{E}+01$ & 1.00E-09 & 5.5 & 0.0422 \\
\hline $\mathrm{C} 28-\mathrm{Fc}$ & $6.08 \mathrm{E}+05$ & $6.72 \mathrm{E}-04$ & 1.11E-09 & 1.1 & 0.0254 \\
\hline $\mathrm{C} 3-\mathrm{Fc}$ & $3.18 \mathrm{E}+05$ & $3.79 \mathrm{E}-04$ & 1.19E-09 & 9.8 & 0.0794 \\
\hline $\mathrm{C} 25-\mathrm{Fc}$ & $2.86 \mathrm{E}+05$ & $5.98 \mathrm{E}-04$ & 2.09E-09 & 3.8 & 0.00962 \\
\hline $\mathrm{C} 30-\mathrm{Fc}$ & 2.07E+05 & 7.37E-04 & 3.57E-09 & 1.5 & 0.0119 \\
\hline $\mathrm{C} 46-\mathrm{Fc}$ & $1.11 \mathrm{E}+05$ & $6.15 \mathrm{E}-04$ & 5.53E-09 & 12.8 & 0.179 \\
\hline $\mathrm{C} 33-\mathrm{Fc}$ & $5.00 \mathrm{E}+05$ & $4.24 \mathrm{E}-06$ & 8.49E-12 & 0.2 & 0.00339 \\
\hline \multicolumn{6}{|c|}{$\begin{array}{l}\text { The values of ka }\left(\mathrm{M}^{-1} \mathrm{~s}^{-1}\right) \text { indicate the binding ability of VHHs-Fc and evinacumab to human } \\
\text { ANGPTL3. The values of kd }\left(\mathrm{M}^{-1} \mathrm{~s}^{-1}\right) \text { indicate the dissociation constant of VHHs-Fc to human } \\
\text { ANGPTL3. The values of } \mathrm{K}_{\mathrm{D}} \text { indicate the affinity ability of VHHs-Fc to human ANGPTL3 and were } \\
\text { calculated by ka/kd value. Rmax (RU) indicates the maximum response value of association. }\end{array}$} \\
\hline
\end{tabular}

\section{Epitope binning assays and affinity test of VHHs-Fc against ANGPTL3}

We next investigated whether the four $\mathrm{VHH}-\mathrm{Fc}$ fusions proteins and evinacumab shared similar antigenbinding epitope. After capturing hANGPTL3 and injecting C27-Fc, fusion proteins C42-Fc and C45-Fc rather than $\mathrm{C} 44-\mathrm{Fc}$ could not be settled. This indicated that not $\mathrm{C} 44-\mathrm{Fc}$, but $\mathrm{C} 42-\mathrm{Fc}$, and $\mathrm{C} 45-\mathrm{Fc}$ fusion protein might share the similar binding epitopes with C27-Fc against hANGPTL3. C27-Fc and C44-Fc fusion proteins were later tested for epitope binning against evinacumab. Our results displayed that after capturing hANGPTL3 and injecting evinacumab, C27-Fc and C44-Fc fusion proteins were all settled (Fig. $2 \mathrm{E})$. These results revealed that $\mathrm{C} 27-\mathrm{Fc}, \mathrm{C} 44-\mathrm{Fc}$ and evinacumab have different hANGPTL3 binding epitopes with each other.

As the CCD and the full-length of ANGPTL3 all can inhibit the LPL activity and to determine the crossing reaction to mouse ANGPTL3, four ANGPTL3 proteins including human CCD of ANGPTL3 (hANGPTL3 (S17-K170)), human full-length of ANGPTL3 (hANGPTL3 (S17-E460)), mouse CCD of ANGPTL3 
(mANGPTL3 (S17-T206)) and mouse full-length of ANGPTL3 (mANGPTL3 (S17-T455)), were used for affinity test of fusion proteins C27-Fc and C44-Fc by SPR. As shown in Figure 3A-H and Table 2, C27-Fc and C44-Fc fusion protein had distinct performance when binding to the four ANGPTL3 proteins. C27-Fc had high affinity to hANGPTL3 (S17-K170) at $27.8 \mathrm{pM}$ with bare dissociation, but it showed a rapid dissociation when binding to hANGPTL3 (S17-E460), mANGPTL3 (S17-T206) and mANGPTL3 (S17T455). C44-Fc had brilliant and expansive affinity to hANGPTL3 (S17-K170) at 0.1909 nM, hANGPTL3 (S17-E460) at $0.1484 \mathrm{nM}, \mathrm{mANGPTL3}$ (S17-T206) at 0.2088 nm and mANGPTL3 (S17-T455) at 0.1402 $\mathrm{nM}$, displaying better affinities comparing with the approved evinacumab. As a result, we identified that C44-Fc might have an expansive affinity to full-length and CCD of both mice and humans ANGPTL3 proteins.

Table 2

Summary of the antibodies affinities against human and mouse ANGPTL3 proteins

\begin{tabular}{|c|c|c|c|c|c|}
\hline Sample & & $k a\left(M^{-1} s^{-1}\right)$ & $k d\left(s^{-1}\right)$ & $K_{\mathrm{D}}(\mathrm{M})$ & $R \max (R U)$ \\
\hline \multirow[t]{3}{*}{ 17-169 hANGPTL3 } & $\mathrm{C} 27-\mathrm{Fc}$ & 7.690E-5 & $1.392 \mathrm{E}-5$ & $1.811 \mathrm{E}-11$ & 207.1 \\
\hline & C44-FC & $5.400 \mathrm{E}-5$ & $1.639 \mathrm{E}-4$ & $3.036 \mathrm{E}-10$ & 37.40 \\
\hline & $\mathrm{EVI}$ & $1.331 \mathrm{E}-5$ & $4.543 \mathrm{E}-4$ & $3.413 \mathrm{E}-10$ & 103.8 \\
\hline \multirow[t]{3}{*}{ 17-460 hANGPTL3 } & C27-Fc & $2.421 \mathrm{E}-5$ & $1.362 \mathrm{E}-3$ & $5.627 \mathrm{E}-9$ & 19.43 \\
\hline & C44-FC & $3.085 \mathrm{E}-6$ & $1.288 \mathrm{E}-3$ & 4.175 E-10 & 23.11 \\
\hline & $\mathrm{EVI}$ & $1.115 \mathrm{E}-5$ & 5.290 E-4 & 4.744 E-10 & 53.40 \\
\hline \multirow[t]{3}{*}{ 17-206 mANGPTL3 } & $\mathrm{C} 27-\mathrm{Fc}$ & $1.575 \mathrm{E}-5$ & $1.292 \mathrm{E}-3$ & 8.202 E-9 & 20.72 \\
\hline & C44-FC & 3.819E-6 & 7.770 E-3 & $2.035 \mathrm{E}-9$ & 48.21 \\
\hline & $\mathrm{EVI}$ & $6.356 \mathrm{E}-5$ & $2.748 \mathrm{E}-4$ & 4.323 E-10 & 190.7 \\
\hline \multirow[t]{3}{*}{ 17-455 mANGPTL3 } & $\mathrm{C} 27-\mathrm{Fc}$ & $7.852 E-5$ & $1.096 \mathrm{E}-3$ & 1.396 E-9 & 9.526 \\
\hline & $\mathrm{C} 44-\mathrm{FC}$ & $1.851 \mathrm{E}-6$ & $1.013 \mathrm{E}-3$ & $5.474 \mathrm{E}-10$ & 49.04 \\
\hline & EVI & $1.249 \mathrm{E}-5$ & $5.603 \mathrm{E}-4$ & $4.486 \mathrm{E}-10$ & 47.88 \\
\hline \multicolumn{6}{|c|}{$\begin{array}{l}\text { The values of } k a\left(\mathrm{M}^{-1} \mathrm{~s}^{-1}\right) \text { indicate the binding ability of } \mathrm{C} 27-\mathrm{Fc}, \mathrm{C} 44-\mathrm{Fc} \text { and evinacumab to human } \\
\text { and mouse ANGPTL3 proteins. The values of } \mathrm{kd}^{-1}\left(\mathrm{M}^{-1} \mathrm{~s}^{-1}\right) \text { indicate the dissociation constant of VHHs- } \\
\text { Fc and evinacumab to ANGPTL3 proteins. The values of } \mathrm{K}_{\mathrm{D}} \text { indicate the affinity ability of VHHs-Fc } \\
\text { and evinacumab to ANGPTL3 proteins and were calculated by ka/kd value. Rmax (RU) indicates the } \\
\text { maximum response value of association. }\end{array}$} \\
\hline
\end{tabular}


The candidates C27-Fc and C44-Fc were analyzed by SEC-HPLC to measure the concentration of fusion protein and its molecular size variations, by nanoDSF to measure thermal stability. As shown in Figure 4A, C44-Fc had a high purity of 100 percent without any aggregation, which was superior to C27-Fc. Meanwhile, C44-Fc and C27-Fc fusion proteins had similar molecular sizes and were smaller than evinacumab. Thermal stability testing revealed that $\mathrm{C} 44-\mathrm{Fc}$ had greater thermal stability than evinacumab and C27-Fc fusion protein due to its higher Tm 1 and Tm 2 (Fig. 4B and Additional file 1: Fig. S3). These findings suggested that C44-Fc fusion protein had better biophysical properties than C27-Fc as a therapeutic antibody.

As the expansive affinity and the better biophysical properties than C27-Fc, C44-Fc was chosen for indepth study. We then investigated the yield, stability via forced degradation and in vitro characterization against ANGPTL3 of C44-Fc. In ExpiCHO cells, the fusion proteins C44-Fc reached its expression level of $500 \mathrm{mg} / \mathrm{L}$, which was roughly twice as high as evinacumab (Fig. 4C). We applied several conditions of forced degradation to test biophysical stability by SEC-HPLC, including low pH ( 5.0), high pH ( 9.0), high temperature of $40^{\circ} \mathrm{C}$ and freeze-thaw. Figure 4D-G shows that all of the monomers were over 97 percentages, indicating that both $\mathrm{C} 44-\mathrm{Fc}$ and evinacumab were exceptionally stable at $\mathrm{pH} 5.0$ and 9.0 for 72 hours, at $40^{\circ} \mathrm{C}$ for 28 days, and after 6 freeze-thaw cycles. We further studied the ability of C44-Fc to block ANGPTL3-mediated suppression of LPL activity by a cell-free LPL assay in vitro. As shown in Figure 5 and Table 3, C44-Fc effectively neutralize the inhibition of LPL activity inducing by four different ANGPTL3s at a concentrated IC $\mathrm{I}_{50}$ values from 1.6 to $5.4 \mathrm{nM}$. As C44-Fc efficaciously neutralized human and mouse CDD or full-length of ANGPTL3 proteins, we employed C57BL/6 mice to assess the following pharmacodynamics study in vivo.

Table 3

$\mathrm{IC}_{50}$ values of C44-Fc blocking ANGPTL3-induced suppression of LPL

\begin{tabular}{|c|c|c|c|c|}
\hline \multirow[t]{3}{*}{ Parameter } & \multicolumn{4}{|l|}{ Protein } \\
\hline & hANGPTL3 & hANGPTL3 & mANGPTL3 (S17-T206) & mANGPTL3 \\
\hline & (S17-K170) & (S17-E460) & & (S17-T455) \\
\hline $\mathrm{C} 44-\mathrm{Fc}, \mathrm{IC}_{50}(\mathrm{nM})$ & 1.6 & 4.6 & 3.6 & 5.4 \\
\hline Evinacumab, $\mathrm{IC}_{50}(\mathrm{nM})$ & 1.7 & 5.4 & 1.8 & 3.7 \\
\hline Control $\mathrm{Ab}, \mathrm{IC}_{50}(\mathrm{nM})$ & NB & NB & NB & NB \\
\hline \multicolumn{5}{|c|}{$\begin{array}{l}\text { The } I_{50} \text { of C44-Fc were measured at ANGPTL3 concentrations of less than } 2.5 \text {-fold } E_{50} \text { values for } \\
\text { ANGPTL3-induced suppression of LPL. }\end{array}$} \\
\hline
\end{tabular}




\section{C44-Fc lowers serum lipids levels in hypercholesterolemic mice}

To investigate the effect of C44-Fc on serum lipid levels in hypercholesterolemic mice, C57BL/ 6 mice were treated by a high-fat/high-cholesterol and cholate diet (HF/HCCD) for four weeks and then treated with a single dose of $25 \mathrm{mg} / \mathrm{kg}$ of isotype control Ab, $10 \mathrm{mg} / \mathrm{kg}$ of C44-Fc, $25 \mathrm{mg} / \mathrm{kg}$ of C44-Fc, or 25 $\mathrm{mg} / \mathrm{kg}$ of evinacumab. Blood samples were acquired on days $0,1,4,7$ and 12 after antibody administration. C44-Fc and evinacumab evoked rapid downward trends in serum TG, TC, and LDL-C levels. On day 4, C44-Fc caused a maximum mean TG and TC level of $44.2 \%$ and $36.6 \%$ reduction in C57BL/6 mice, respectively, when compared to isotype controls. Besides, the mice treated with evinacumab exhibited $44.9 \%$ and $38.9 \%$ reduction of TG and TC level, respectively, which shows no significance with our anti-ANGPTL3 VHH antibody candidate, C44-Fc. During days 4 7 post antibody administration, LDL-C levels in C44-Fc and evinacumab-injected mice were reduced over $54.4 \%$. Furthermore, the hypercholesterolemic mice receiving a single dose of $\mathrm{C} 44-\mathrm{Fc}$ and evinacumab treatment still exhibited considerably lower TC and LDL-C levels on day 12 (the last day of the trial).

We ulteriorly confirmed LPL activity in hypercholesterolemic mice after C44-Fc treatment. Our results showed that the effect of $\mathrm{C} 44-\mathrm{Fc}$ on the serum lipids reduction was closely related with the upregulated activity levels of plasma LPL activity after intraperitoneally injecting heparin (Fig. 6G). The facilitation of TG clearance triggered by C44-Fc was further assessed by fat tolerance tests. After injection of intralipid, plasma $\mathrm{TG}$ of mice treated with control $\mathrm{Ab}$ had a remarkable increase which was significantly attenuated by C44-Fc, indicating C44-Fc administration remarkably improved fat tolerance in HF/HCCD-fed mice.

\section{Multiple administration of C44-Fc relieves hepatic steatosis induced by HF/HCCD feeding}

The therapeutic effect of C44-Fc on NAFLD was measured in a HF/HCCD-fed mice. After an 8-week feed of C57BL/6 mice with HF/HCCD, mice showed an increase in plasma TC and LDL-C levels from 4.3 to $10.6 \mathrm{mmol} / \mathrm{L}$ and from 0.41 to $2.3 \mathrm{mmol} / \mathrm{L}$, respectively. During the HF/HCCD diet, mice were given isotype control $\mathrm{Ab}(25 \mathrm{mg} / \mathrm{kg}), \mathrm{C} 44-\mathrm{Fc}(25 \mathrm{mg} / \mathrm{kg})$, or evinacumab $(25 \mathrm{mg} / \mathrm{kg})$ once a week for 6 weeks (Fig. 7A). Blood samples were collected after a 4-hour fast on day 4 after each injection. Compared to the chow-diet mice, HF/HCCD mice had increase in liver weight ( 1.7 fold), liver TG contents $(\sim 1.6$ fold) and hepatic lipid accumulation, indicating the pathological changes of NAFLD in HF/HCCD mice. ${ }^{36,37}$ The treatments of C44-Fc (red lines) and evinacumab (blue lines) decreased plasma TG, TC and LDL-C markedly and sustainably in comparison to isotype control Ab (Fig. 7). After the first injection of C44-Fc, a significant decline trends in TG (from $0.95 \mathrm{mmol} / \mathrm{L}$ to $0.59 \mathrm{mmol} / \mathrm{L}), \mathrm{TC}(\sim 5.9 \mathrm{mmol} / \mathrm{L})$ and LDL-C ( 1.7 $\mathrm{mmol} / \mathrm{L})$ could be found after 1 day post C44-Fc administration. Similarly, there were also noticeable reductions in TG (from $0.95 \mathrm{mmol} / \mathrm{L}$ to $0.55 \mathrm{mmol} / \mathrm{L}), \mathrm{TC}(\sim 7.3 \mathrm{mmol} / \mathrm{L})$ and LDL-C ( 2.0 mmol/L) after the first administration of evinacumab. 
After 6 weeks of C44-Fc treatment, liver overweight of the HF/HCCD mice was significantly alleviated, with liver coloration and morphology ameliorated (Fig. 8B-C). Fairly, $25 \mathrm{mg} / \mathrm{kg}$ of C44-Fc treatment had no significant effects on weights and TG contents of epididymal fat or heart, insulin tolerance test (ITT) and glucose tolerance test (GTT) (Additional file 1: Fig. S4 and S5). Furthermore, our results also demonstrated that HF/HCCD feeding led to a hepatic lipid accumulation (Fig. 8D-E) as well as increased level of ALT and AST, which was distinctly ameliorated by weekly C44-Fc (Fig. 8F). Collectively, these findings suggested that $\mathrm{C} 44-\mathrm{Fc}$ could reduce serum lipids levels and relieves hepatic steatosis induced by HF/HCCD feeding.

\section{Discussion}

NAFLD is a metabolic disease and positively associated with hypercholesterolemia that is increasingly acknowledged as the key factor of excessive lipid uptake and lipotoxicity in liver resulting in pathologic insult. ${ }^{3,41}$ It is projected to develop into cirrhosis with increasing risk of hepatocellular carcinoma and may be the main inducement of liver transplantation..$^{42}$ Despite its prevalence and seriousness, NAFLD still lacks effective therapeutics. ${ }^{38}$ ANGPTL3 is a key lipid metabolism regulator leading to high levels of plasma TG, TC and LDL-C. ${ }^{10}$ In this study, we investigated whether inhibiting ANGPTL3 is an effective approach for NAFLD treatment. Herein, we have developed a VHH-Fc fusion protein against ANGPTL3, which significantly ameliorated hepatic lipid accumulation and liver injury in HF/HCCD-induced NAFLD mice through reducing serum lipid levels of TG, TC and LDL-C.

Although NAFLD was availably treated by reducing blood lipids and blood glucose, anti-fibrosis and antiinflammatory in animal models, it still affects approximately $25 \%$ of the population worldwide. ${ }^{39}$ Until now, exclusive Saroglitazar was the approved drug for the treatment of NAFLD, which is a PPAR- $a / Y$ agonist. ${ }^{7}$ Unfortunately, the PPAR-a/y agonist has been reported to increase about $2-4 \%$ of body weight and to have adverse reactions including peripheral oedema precipitated in probably $5 \%$ of treated patients and fatal heart failure occurred in approximately $11 \%$ of treated patients. ${ }^{40}$ The traditional lipidsreducing agents statins were not applicable to NAFLD treatment as for its intolerance or side effects. ${ }^{41}$ Thus, a safe and efficient targets for NAFLD is urgently needed. ANGPTL3, as a depressor of LPL and EL, was recently revealed elevated expression levels in liver of NAFLD patients and contributed to increased circulating ANGPTL3. ${ }^{16}$ Interestingly, neither the homozygous loss-of-function of ANGPTL3 nor the inhibitors of ANGPTL3 all did generate adverse effects with preeminent safety and tolerability, but leaded to very low TG, VLDL, LDL and non-esterified fatty acids (NEFAs). ${ }^{42,43}$ Our results suggested ANGPTL3 inhibitor showed splendid reduction of TG (44.2\%), TC (36.6\%) and LDL-C (54.4\%). More importantly, its multiple administrations significantly reduced $18.3 \%$ of liver weight, ameliorated liver coloration and morphology, improved hepatic lipid accumulation and protected HF/HCCD-related liver injury. All those results indicated blocking ANGPTL3 was a novel therapeutic approaches for NAFLD with satisfactory efficacy and safety. 
The existing ANGPTL3 inhibiting tactics includes the monoclonal antibody (evinacumab), ASO (IONISANGPTL3-LRx) and the CRISPR/Cas9 gene knockout technology, whereas neither of them is employed for NAFLD therapy. Evinacumab and the ASO-based drugs are expensive and the CRISPR/Cas9 gene knockout technology haven't been approved for in clinical use. ${ }^{19-21}$ Nanobodies, which are promising therapeutic agents, display higher binding ability, better penetration and superior stability. Recently, caplacizumab, which is a bivalent $\mathrm{VHH}$ for thrombotic thrombocytopenic purpura treatment, has been regarded as the first nanobody approved by the FDA. ${ }^{35,44,45}$ The development and characterization of VHHs that specifically block ANGPTL3 have not been reported so far. Hence, we developed a nanobody as the ANGPTL3 inhibitor by establishing a naïve phage-display VHHs library about $5 \times 10^{9}$ after immunizing alpaca with hANGPTL3 (S17-K170). We obtained 11 specific VHH candidates against hANGPTL3 (S17-K170) with different CDRs sequences by indirect ELISA from the library.

The VHHs have prominently short circulation half-life due to its small size of approximately $15 \mathrm{kDa}$ and is restricted to disease therapy. Multiple methods have been applied to benefit its half-life period in vivo, such as VHHs can be conjugated with human serum albumin, fused to IgG-Fc and PEGylated. ${ }^{46-48}$ Meanwhile, previous studies revealed that bivalent nanobody had better affinity than monovalent modality. In particular, the bivalent VHHs fused with Fc showed above 10 times higher affinity and stronger therapeutic effects than monovalent in vitro and in vivo, which also emerged longer half-life of about 15 days when compared to that of monovalent ( 30 $\mathrm{min}$ ) and tandem bivalent VHHs ( 60 min). ${ }^{48 \text {, }}$ ${ }^{49}$ In this study, the 11 candidate VHHs were fused to human IgG1 Fc domain to construct the bivalent VHHs-Fc fusion proteins and then successfully expressed in ExpiCHO cells, followed by its purification by protein A affinity chromatography. The pharmacodynamics results of single administration suggests that the VHHs-Fc fusion protein has long half-life in vivo as the durable lipid-reducing effect of 12 days.

The full length and the cleaved CCD of ANGPTL3 proteins all can regulate lipid metabolism by inhibiting the enzyme activity of LPL and EL. ${ }^{8}$ Although C27-Fc had $~ 12$ times higher affinity to the cleaved CCD of hANGPTL3 than C44-Fc, its affinity against full length of human and mouse ANGPTL3 proteins remained unsatisfactory. Thus, we ultimately selected C44-Fc for further study its therapeutic effects on NAFLD due to its high affinity and LPL de-repression of both the human full length and CCD of ANGPTL3 along with crossing reaction to mouse ANGPTL3s. The affinities against human and mouse ANGPTL3 proteins of C44-Fc were about $1.7 \sim 3.2$ times higher than evinacumab. But in terms of $\mathrm{IC}_{50}$ of rescuing ANGPTL3smediated suppression of LPL activity and the lipid-reducing effect as well as relieving function in NAFLD, C44-Fc performed no significant difference with evinacumab. This is probably because of the different ANGPTL3-binding epitopes between C44-Fc and evinacumab.

In addition to its high affinity, the C44-Fc had many merits in the discovery and development process as a therapeutic antibody compared to evinacumab. The productivity of Camelidae antibody C44-Fc in ExpiCHO cells reached $\sim 500 \mathrm{mg} / \mathrm{L}$ that was twice as much as evinacumab and isotype control human IgG1. Besides, previous research reported nanobodies had excellent thermostability and chemostability, ${ }^{28,}$ 50 likewise, we detected the thermal stability and the forced degradation of C44-Fc. The results notarized 
nanobody $(\mathrm{C} 44-\mathrm{Fc})$ had a better thermostability $\left(\operatorname{Tm} 159.7^{\circ} \mathrm{C}\right)$ than evinacumab $\left(\operatorname{Tm} 157.3^{\circ} \mathrm{C}\right)$ and had no obvious degradation or aggregation at low $\mathrm{pH}$, high $\mathrm{pH}$, high temperature $\left(40^{\circ} \mathrm{C}\right.$ for 28 days) and freezethaw. All these results indicated that $\mathrm{C} 44-\mathrm{Fc}$ could decrease the expenditure of production, storage and transportation in the future development.

Consistent with those results, we had developed a VHH-Fc fusion protein as the ANGPTL3 inhibitor that showed remarkable lipid-lowering and therapeutical effect on HF/HCCD-induced NAFLD mice, which indicated the C44-Fc fusion protein was likely to be a prospective curative agent for hypercholesterolemic and NAFLD patients. It also might be a latent treatment in other metabolic disorders such as cardiovascular diseases. Nonetheless, further research such as humanization, pharmacodynamics study in cynomolgus monkeys can be performed to develop it into a therapeutic drug.

\section{Conclusions}

In this work, we demonstrated that blocking ANGPTL3 could relieve lipid accumulation of liver and hepatic injury in HF/HCCD -induced NAFLD mice. We screened out and developed a novel VHH-Fc fusion protein (C44-Fc) against human and mouse ANGPTL3 that owns different binding epitopes with evinacumab. This camelid anti-ANGPTL3 VHH protein showed better affinity, higher productivity and excellent stability. We further illustrated its inhibiting effect on ANGPTL3-mediated LPL activity blockade in vitro, lipid-reducing function in hypercholesterolemic mice, as well as ameliorating efficacy on hepatic lipid accumulation and liver injury in NAFLD mice, indicating that targeting ANGPTL3 is a prospective therapeutic approach for NAFLD.

\section{Methods}

\section{Immunization and library generation}

Alpaca immunizations and the following construction of $\mathrm{VHH}$ library were performed as reported previously. ${ }^{50} \mathrm{~A}$ female alpaca was subcutaneously injected with $500 \mu \mathrm{g}$ of emulsified hANGPTL3(17170)-mFc antigen (fused from human S17-K170 ANGPTL3 and mouse IgG1-Fc and expressed by our laboratory) on days 1, 21, 42, 63. Blood was collected at 7 days after the last three immunizations for the isolation of peripheral blood lymphocytes (PBLs) by density gradient centrifugation using ficoll-paque plus (Cytiva, USA, Cat\# 17144002). The total RNA of PBLs was extracted by Trizol, followed by a RT-PCR for amplification of the coding sequences of the anti-ANGPTL3 VHH antibody candidates. Next, the VHHs library was generated by electro-transforming the VHH genes and pHEN1 phagemid recombinant vector into competent TG1 E.coli. Finally, the VHHs library was infected with M13KO7 helper phages (Thermo Scientific Ltd, USA, Cat\# 18311019) to construct the phagemid VHH-presenting library.

\section{hANGPTL3-specific VHHs enrichment}

Phages presenting VHHs with hANGPTL3-specific binding were enriched after three rounds of screening on hANGPTL3 (S17-220P)-His-immobilized (Novoprotein, China, Cat\# Q9Y5C1) 96-well plates. The 
concentrations of antigen coated on every well for three rounds were decreased in the order of $5,2,1 \mu \mathrm{g}$ per well and simultaneously a blank well was employed as a negative control. After washing with PBST for three times, all wells were blocked in a $37^{\circ} \mathrm{C}$ constant temperature incubator for $1 \mathrm{~h}$ with PBS containing $3 \%(\mathrm{w} / \mathrm{v})$ milk powder and washed with PBS for 3 times. Approximately $10^{11}$ phages (diluted with PBS containing $3 \%(\mathrm{w} / \mathrm{v})$ milk powder) were added to the antigen-coated plates and then put into a $37^{\circ} \mathrm{C}$ constant temperature incubator for $1 \mathrm{~h}$. After washing with PBST (containing $0.05 \%$ Tween 20 ) for 6 times in the first round and 8 times in the remaining two panning rounds, hANGPTL3-specific phages were retained and added into TG1 E. coli culture to amplify at $30^{\circ} \mathrm{C}$ with shaking and rescued with $\mathrm{M} 13 \mathrm{KO} 7$ helper phages at $30^{\circ} \mathrm{C}$ overnight. The purified phages were precipitated by $\mathrm{PEG} 8,000 / \mathrm{NaCl}$ for the subsequent panning round. For each bio-panning, enrichment was performed as described above and was evaluated by the bacteria population which were infected with the purified phages and then cultured on LB agar plates with ampicillin.

\section{Indirect ELISA Screening}

ELISA was employed to evaluate the affinity of phage-displayed VHH to ANGPTL3. Forty-eight individual bacteriophages were selected from the second and third bio-panning LB agar plates and rescued with $\mathrm{M} 13 \mathrm{KO} 7$ helper phages at $30^{\circ} \mathrm{C}$ overnight. Then, the viral supernatants containing VHHs were added into the plates coated with hANGPTL3 (S17-220P)-His protein, and PBS containing $3 \%(\mathrm{w} / \mathrm{v})$ milk powder was used as a negative control. After washing with PBST (containing 0.05\% Tween 20) for 6 times, the bound VHHs were determined by HRP-conjugated rabbit anti-M13 antibody (1:500, Abcam, USA) that could react with TMB (Beyotime, China, P0209) followed by the OD450 value testing. Finally, the bacteriophages with OD450 value 5 -fold higher than the negative control were regarded as positive clones and used for subsequent sequencing.

\section{VHHs-Fc expression and purification}

The encoding sequences of 11 nanobodies fused to human IgG1-Fc were synthesized and inserted into pTT5 vector. The DNA sequences encoding the variable regions of evinacumab were cloned and digested into corresponding regions of human IgG1 on pTT5 vector by EcoRI and Nhel (Takara, Biotechnology, Otsu, Japan). All VHHs-Fc and evinacumab expression plasmids were transfected into ExpiCHO-S cells (Gibco, Thermo Scientific Ltd.) by $\mathrm{CHO}$ Transfection Kit and cultured in ExpiCHO expression media (Gibco, Thermo Scientific Ltd.) according to its Max Titer protocol. The culture supernatants were collected 12 days after transfection and then purified by HiTrap MabSelect Prism A column (GE Healthcare).

\section{Kinetic screening ANGPTL3-specific VHHs-Fc and Epitope binding test}

The surface plasmon resonance (SPR) was employed for kinetic screening on a Biacore T200 (GE Healthcare, USA). The human IgG antibody was immobilized on a CM5 chip (GE Healthcare, USA) by the human antibody capture kits (Cytiva, USA), and the $11 \mathrm{VHH}-\mathrm{Fc}$ fusion proteins were then respectively captured on the chip at about $200 \mathrm{RU}$ through modulating the time of capturing. Subsequently, 0.05 
$\mathrm{mg} / \mathrm{mL}$ of hANGPTL3(17-170)-mFc was flowed over the chip at $100 \mathrm{~s}$ for binding and $600 \mathrm{~s}$ for dissociation. Then, the regeneration buffer of $\mathrm{pH} 2.5 \mathrm{Glycine}-\mathrm{HCl}$ (GE Healthcare, USA) was flowed over the chip for $30 \mathrm{~s}$ before the next round. The KD value of each VHH-Fc indicated the affinity to hANGPTL3.

The mouse antibody capture kit (Cytiva, USA) was used to immobilize mouse IgG antibody on a CM5 chip (GE healthcare, USA) and then the antigen hANGPTL3(17-170)-mFc was captured on the chip at about $160 \mathrm{RU}$. Next, the C27-Fc was injected into the chip for 120s to occupy all the C27-Fc epitopes on the hANGPTL3, then the other fusion proteins C42-Fc, C45-Fc and C44-Fc were injected into the chip for 120s to test whether they have similar antigen-binding epitope with $\mathrm{C} 27-\mathrm{Fc}$. A rise in curve, meant the antigenbinding epitope with $\mathrm{C} 27-\mathrm{Fc}$ was different. Likewise, the $\mathrm{C} 27-\mathrm{Fc}$ and $\mathrm{C} 44-\mathrm{Fc}$ were tested with evinacumab using the above method.

\section{Affinity determination}

The affinities of VHHs-Fc binding to hANGPTL3 (S17-K170)-mFc, hANGPTL3 (S17-E460)-His10, mANGPTL3 (S17-T206)-His6 and mANGPTL3 (S17-T455)-His10 were determined by SPR. The human IgG antibody was immobilized on a CM5 chip, then The VHHs-Fc were captured on the chip at about 200 RU. Next, the hANGPTL3(17-170)-mFc was diluted by EP buffer at concentrations of $0.3125,0,625,1.25$, 2.5, 5, 10 and $20 \mathrm{nM}$ and flowed over the chip for $120 \mathrm{~s}$ for binding and $480 \mathrm{~s}$ for dissociation. As for hANGPTL3 (S17-E460)-His10, mANGPTL3 (S17-T206)-His6 and mANGPTL3 (S17-T455)-His10, were immobilized on a CM5 chip at first and then the VHHs-Fc were flowed over the chip at $120 \mathrm{~s}$ for binding and $480 \mathrm{~s}$ for dissociation after dilution by EP buffer.

\section{SEC-HPLC for biophysical stability evaluation}

SEC-HPLC was performed to assess the concentration of VHHs-Fc using an Agilent 1260 Infinity II SFC System with TOSOH TSKgel G3000WXL column $(7.8 \mathrm{~mm} \times 30 \mathrm{~cm}, 5 \mu \mathrm{m})$ at $1.0 \mathrm{~mL} / \mathrm{min}$ flow rate of PBS. Each sample was injected with $100 \mu \mathrm{g}$ of protein and measured by UV detection for $30 \mathrm{~min}$ at $280 \mathrm{~nm}$ and $37^{\circ} \mathrm{C}$.

\section{Thermal Stability Analysis}

The thermal stabilities of VHHs-Fc were evaluated by NanoTemper PR.48 (NanoTempe Scientific, Germany) through monitoring the fluorescence of tryptophan residues excited at a temperature gradient of $20^{\circ} \mathrm{C}$.

In vitro LPL assay

The ability of C44-Fc to block ANGPTL3-mediated suppression of LPL activity was tested by a cell-free LPL assay in vitro. Four kinds of ANGPTL3 proteins included hANGPTL3 (S17-K170)-mFc, hANGPTL3 (S17-E460)-His10, mANGPTL3 (S17-T206)-His6 and mANGPTL3 (S17-T455)-His10 or with C44-Fc, evinacumab or control antibody (Ab) were pre-incubated with $40 \mathrm{nM}$ of bovine LPL (Sigma, USA, 9004-028) and $0.46 \mu \mathrm{M}$ of human ApoCII (EMD Biosciences, San Diego, CA), and then were measured by LPL 
Activity Fluorometric Assay Kit (Biovision, USA) at $37^{\circ} \mathrm{C}$ for $1 \mathrm{~h}$. Fluorescence was tested on a BioTek Multi-Mode Microplate Reader at 482/515 nm (excitation/emission).

\section{Animal Study}

C57BL/6 mice (male, 5-6 weeks old) were obtained from Shanghai SIPPR-BK Laboratory Animal Co. Ltd. (Shanghai, China) and were kept under the standard specific pathogen-free (SPF) environment. Mice were supplied with high-fat/high-cholesterol and cholate diet (HF/HCCD, Teklad TD.90221, which contains $15.8 \%$ fat, $1.25 \%$ cholesterol and $0.5 \%$ sodium cholate) or chow diet after being acclimated to experiment environment for 1 week. ${ }^{51}$ For single administration studies, C57BL/6 mice were fed with HF/HCCD for four weeks and then treated with subcutaneous injection of control Ab or C44-Fc. Blood samples were collected before antibody injection as a baseline and 1, 4, 7 and 12 days later by retro-orbital bleed after a $4 \mathrm{~h}$ fast. For the Postheparin plasma LPL activity, mice were treated with heparin (100 U/kg) intraperitoneally injected in tail and 5 min later plasma samples were collected into EDTA-coated tubes which were preserved on ice. As for multiple administration, C57BL/6 mice were fed with HF/HCCD for eight weeks and then treated with subcutaneous injection of control Ab or C44-Fc weekly for six weeks. Blood samples were collected four days after each injection. At the end of the experiment, plasma lipoproteins were separated as described previously. ${ }^{52}$

\section{Metabolic Analysis}

Lipid tolerance test (LTT), insulin tolerance test (ITT), and glucose tolerance test (GTT) were performed 4 days after antibody injection (25 mg/kg). For LTT, C57BL/6 mice were injected intraperitoneally with 10 $\mu \mathrm{g} / \mathrm{kg}$ body weight of intralipid (Baxter Healthcare Corporation, USA) after a 2-hour fast. For ITT, mice were intraperitoneally injected with $1 \mathrm{IU}$ insulin (Beyotime, Shanghai) per kg body weight after a 4-hour fast. For GTT, mice were intraperitoneally injected with $1 \mathrm{~g} / \mathrm{kg}$ body weight of glucose (Beyotime, Shanghai) after 6 hours of fasting. All blood samples were acquired at 0, 20, 40, 60, 120 or 240 min after administration.

\section{Biochemistry Analysis}

All serum levels of TG, TC, LDL-C, alanine aminotransferase (ALT), aspartate aminotransferase (AST) and tissue lipid content were detected using commercial kits (Nanjing Jiancheng Bioengineering, Nanjing). The blood glucose levels were tested by blood glucose test strips (Roche, Germany).

\section{Histopathological study}

The liver samples were weighed and fixed with 4\% paraformaldehyde. Subsequently, all liver samples were embedded with paraffin and stained with hematoxylin and eosin (H\&E) or embedded in OTC and freezed for oil red 0 staining.

\section{Data analyses}

All data were presented as mean \pm SEM. The significant difference between groups were operated using Student's t-test or one-way ANOVA and were indicated + , $\S$ or * as $P<0.05$, ††, §§ or ** as $P<0.01$ and 
$\S \S \S$ or *** as $\mathrm{P}<0.001$.

\section{Abbreviations}

ALT, alanine aminotransferase; ANGPTL3, Angiopoietin-like protein 3; AST, aspartate transaminase; ASO, antisense oligonucleotide; CCD, coiled-coil domain; EL, endothelial lipase; ELISA, Enzyme-linked immunosorbent assay; Fc, The constant region fragment of the human immunoglobulin gamma; $\mathrm{FH}$, familial hypercholesterolemia; FLD, fibrinogen-like domain; GTT, glucose tolerance test; H\&E, hematoxylin and eosin; HF/HCCD, high-fat/high-cholesterol and cholate diet; IgG: Immunoglobulin gamma; ITT, insulin tolerance test; LDL: Low density lipoprotein; LDL-C: Low density lipoprotein cholesterol; LDLR: Low density lipoprotein receptor; LPL, lipoprotein lipase; LTT, Lipid tolerance test; LXRs, liver X receptors; OD450: The optical density value at $450 \mathrm{~nm}$; PBLs, peripheral blood lymphocytes; PBST: Phosphate buffer solution with $0.05 \%$ tween 20 ; NAFLD, Non-alcoholic fatty liver disease; RFU: The relative fluorescence unit; RU: Response units; sdAb: Single domain antibody; SPR: Surface plasmon resonance; TC, Total cholesterol; TES buffer: 0.2 M Tris- $\mathrm{HCl}$ pH 8.0, 0.5 mM EDTA, 0.5 M sucrose; TG, triglyceride; $\mathrm{VHH}$ : High variable region of the heavy chain antibody; kDa: Kilo Dalton.

\section{Declarations}

\section{Acknowledgements}

Not applicable.

\section{Contributions}

Dianwen Ju and Jiajun Fan designed the research; Xiaozhi Hu, Lei Han and Zhonglian Cao carried out experiments; Xiaozhi Hu, Jiajun Fan and Qianqian Ma analyzed the data and write the manuscript; Caili $\mathrm{Xu}$, Jingyun Luan and Yanyang Nan draw the figures; Tao Wu, Yuting Zhang, Hanqi Wang revised the manuscript writing; all authors approved the final version of the manuscript.

\section{Funding}

This study was supported by grants from the National Natural Science Foundation of China (Grant Nos. $81803529,81773620,82073752)$ and the Shanghai Science and Technology Fund (20JC1411000, 20S11904700 and 21QB1401800).

\section{Availability of data and materials}

All data generated or analyzed during this study are included in the article.

\section{Ethics approval and consent to participate}

Animal studies were approved by the Ethics Committee of School of Pharmacy, Fudan University. 


\section{Competing Interests}

The authors declare no conflict of interest.

\section{Consent for publication}

Not applicable.

\section{Author details}

${ }^{1}$ School of Pharmacy \& Minhang Hospital, Shanghai Engineering Research Center of Immunotherapeutics, Fudan University, Shanghai, 201203, China. ${ }^{2}$ Children's Hospital of Fudan University, National Children's Medical Center, Shanghai 201203, China. ${ }^{3}$ Ben May Department of Cancer Research, The University of Chicago, Chicago, IL, 60615, USA.

\section{References}

1. Sinha RA, Bruinstroop E, Singh BK, Yen PM. Nonalcoholic Fatty Liver Disease and Hypercholesterolemia: Roles of Thyroid Hormones, Metabolites, and Agonists. Thyroid. 2019;29:1173-91.

2. Hegele RA. Plasma lipoproteins: genetic influences and clinical implications. Nat Rev Genet. 2009;10:109-21.

3. Samuel VT, Shulman GI. Nonalcoholic Fatty Liver Disease as a Nexus of Metabolic and Hepatic Diseases. Cell Metab. 2018;27:22-41.

4. Eslam M, Valenti L, Romeo S. Genetics and epigenetics of NAFLD and NASH: Clinical impact. J Hepatol. 2018;68:268-79.

5. Zhou J, Zhou F, Wang W, Zhang XJ, Ji YX, Zhang P, She ZG, Zhu L, Cai J, Li H. Epidemiological Features of NAFLD From 1999 to 2018 in China. Hepatology. 2020;71:1851-64.

6. Li W, Liu J, Cai J, Zhang XJ, Zhang P, She ZG, Chen S, Li H. NAFLD as a continuous driver in the whole spectrum of vascular disease. J Mol Cell Cardiol. 2021;163:118-32.

7. Gawrieh S, Noureddin M, Loo N, Mohseni R, Awasty V, Cusi K, Kowdley KV, Lai M, Schiff E, Parmar D. Patel P and Chalasani N. Saroglitazar, a PPAR-a/Y Agonist, for Treatment of NAFLD: A Randomized Controlled Double-Blind Phase 2 Trial. Hepatology. 2021;74:1809-24.

8. Ono M, Shimizugawa T, Shimamura M, Yoshida K, Noji-Sakikawa C, Ando Y, Koishi R, Furukawa H. Protein region important for regulation of lipid metabolism in angiopoietin-like 3 (ANGPTL3): ANGPTL3 is cleaved and activated in vivo. J Biol Chem. 2003;278:41804-9.

9. Camenisch G, Pisabarro MT, Sherman D, Kowalski J, Nagel M, Hass P, Xie MH, Gurney A, Bodary S, Liang XH, Clark K, Beresini M, Ferrara N, Gerber HP. ANGPTL3 stimulates endothelial cell adhesion and migration via integrin alpha vbeta 3 and induces blood vessel formation in vivo. J Biol Chem. 2002;277:17281-90. 
10. Romeo S, Yin W, Kozlitina J, Pennacchio LA, Boerwinkle E, Hobbs HH, Cohen JC. Rare loss-offunction mutations in ANGPTL family members contribute to plasma triglyceride levels in humans. J Clin Invest. 2009;119:70-9.

11. Lee EC, Desai U, Gololobov G, Hong S, Feng X, Yu XC, Gay J, Wilganowski N, Gao C, Du LL, Chen J, Hu Y, Zhao S, Kirkpatrick L, Schneider M, Zambrowicz BP, Landes G, Powell DR, Sonnenburg WK. Identification of a new functional domain in angiopoietin-like 3 (ANGPTL3) and angiopoietin-like 4 (ANGPTL4) involved in binding and inhibition of lipoprotein lipase (LPL). J Biol Chem. 2009;284:13735-45.

12. Minicocci I, Montali A, Robciuc MR, Quagliarini F, Censi V, Labbadia G, Gabiati C, Pigna G, Sepe ML, Pannozzo F, Lütjohann D, Fazio S, Jauhiainen M, Ehnholm C, Arca M. Mutations in the ANGPTL3 gene and familial combined hypolipidemia: a clinical and biochemical characterization. J Clin Endocrinol Metab. 2012;97:E1266-75.

13. Arca M, D'Erasmo L, Minicocci I. Familial combined hypolipidemia: angiopoietin-like protein-3 deficiency. Curr Opin Lipidol. 2020;31:41-8.

14. Kaplan R, Zhang T, Hernandez M, Gan FX, Wright SD, Waters MG, Cai TQ. Regulation of the angiopoietin-like protein 3 gene by LXR. J Lipid Res. 2003;44:136-43.

15. Inaba T, Matsuda M, Shimamura M, Takei N, Terasaka N, Ando Y, Yasumo H, Koishi R. Makishima M and Shimomura I. Angiopoietin-like protein 3 mediates hypertriglyceridemia induced by the liver $\mathrm{X}$ receptor. J Biol Chem. 2003;278:21344-51.

16. Barchetta I, Cimini FA, Chiappetta C, Bertoccini L, Ceccarelli V, Capoccia D, Gaggini M, Di Cristofano C, Della Rocca C, Silecchia G, Leonetti F, Lenzi A, Gastaldelli A, Cavallo MG. Relationship between hepatic and systemic angiopoietin-like 3 , hepatic Vitamin D receptor expression and NAFLD in obesity. Liver Int. 2020;40:2139-47.

17. Windler E, Nitschmann S. [Evinacumab in patients with treatment-refractory hypercholesterolemia]. Internist (Berl). 2021;62:686-9.

18. Dewey FE, Gusarova V, Dunbar RL, O'Dushlaine C, Schurmann C, Gottesman O, McCarthy S, Van Hout CV, Bruse S, Dansky HM, Leader JB, Murray MF, Ritchie MD, Kirchner HL, Habegger L, Lopez A, Penn J, Zhao A, Shao W, Stahl N, Murphy AJ, Hamon S, Bouzelmat A, Zhang R, Shumel B, Pordy R, Gipe D, Herman GA, Sheu WHH, Lee IT, Liang KW, Guo X, Rotter JI, Chen YI, Kraus WE, Shah SH, Damrauer S, Small A, Rader DJ, Wulff AB, Nordestgaard BG, Tybjærg-Hansen A, van den Hoek AM, Princen HMG, Ledbetter DH, Carey DJ, Overton JD, Reid JG, Sasiela WJ, Banerjee P, Shuldiner AR, Borecki IB, Teslovich TM, Yancopoulos GD, Mellis SJ. Gromada J and Baras A. Genetic and Pharmacologic Inactivation of ANGPTL3 and Cardiovascular Disease. N Engl J Med. 2017;377:211-21.

19. Qiu M, Glass Z, Chen J, Haas M, Jin X, Zhao X, Rui X, Ye Z, Li Y, Zhang F, Xu Q. Lipid nanoparticlemediated codelivery of Cas 9 mRNA and single-guide RNA achieves liver-specific in vivo genome editing of Angptl3. Proc Natl Acad Sci U S A. 2021;118.

20. Graham MJ, Lee RG, Brandt TA, Tai LJ, Fu W, Peralta R, Yu R, Hurh E, Paz E, McEvoy BW, Baker BF, Pham NC, Digenio A, Hughes SG, Geary RS, Witztum JL, Crooke RM, Tsimikas S. Cardiovascular and 
Metabolic Effects of ANGPTL3 Antisense Oligonucleotides. N Engl J Med. 2017;377:222-32.

21. Dewey FE, Gusarova V, Dunbar RL, O'Dushlaine C, Schurmann C, Gottesman O, McCarthy S, Van Hout CV, Bruse S, Dansky HM, Leader JB, Murray MF, Ritchie MD, Kirchner HL, Habegger L, Lopez A, Penn J, Zhao A, Shao W, Stahl N, Murphy AJ, Hamon S, Bouzelmat A, Zhang R, Shumel B, Pordy R, Gipe D, Herman GA, Sheu WHH, Lee IT, Liang KW, Guo X, Rotter JI, Chen YI, Kraus WE, Shah SH, Damrauer S, Small A, Rader DJ, Wulff AB, Nordestgaard BG, Tybjaerg-Hansen A, van den Hoek AM, Princen HMG, Ledbetter DH, Carey DJ, Overton JD, Reid JG, Sasiela WJ, Banerjee P, Shuldiner AR, Borecki IB, Teslovich TM, Yancopoulos GD, Mellis SJ. Gromada J and Baras A. Genetic and Pharmacologic Inactivation of ANGPTL3 and Cardiovascular Disease. N Engl J Med. 2017;377:211-21.

22. Vermersch E, Jouve C, Hulot JS. CRISPR/Cas9 gene-editing strategies in cardiovascular cells. Cardiovasc Res. 2020;116:894-907.

23. Tanaka M, Nyce JW. Respirable antisense oligonucleotides: a new drug class for respiratory disease. Respir Res. 2001;2:5-9.

24. Conrath KE, Wernery U, Muyldermans S, Nguyen VK. Emergence and evolution of functional heavychain antibodies in Camelidae. Dev Comp Immunol. 2003;27:87-103.

25. Hamers-Casterman C, Atarhouch T, Muyldermans S, Robinson G, Hamers C, Songa EB, Bendahman N, Hamers R. Naturally occurring antibodies devoid of light chains. Nature. 1993;363:446-8.

26. Riechmann L, Muyldermans S. Single domain antibodies: comparison of camel VH and camelised human VH domains. J Immunol Methods. 1999;231:25-38.

27. Arbabi-Ghahroudi M, Tanha J, MacKenzie R. Prokaryotic expression of antibodies. Cancer Metastasis Rev. 2005;24:501-19.

28. Li X, Duan X, Yang K, Zhang W, Zhang C, Fu L, Ren Z, Wang C, Wu J, Lu R, Ye Y, He M, Nie C, Yang N, Wang J, Yang H, Liu X, Tan W. Comparative Analysis of Immune Repertoires between Bactrian Camel's Conventional and Heavy-Chain Antibodies. PLoS One. 2016;11:e0161801.

29. Pothin E, Lesuisse D, Lafaye P. Brain Delivery of Single-Domain Antibodies: A Focus on VHH and VNAR. Pharmaceutics. 2020;12.

30. De Vlieger D, Ballegeer M, Rossey I, Schepens B, Saelens X. Single-Domain Antibodies and Their Formatting to Combat Viral Infections. Antibodies (Basel). 2018;8.

31. Dumoulin M, Conrath K, Van Meirhaeghe A, Meersman F, Heremans K, Frenken LG, Muyldermans S, Wyns L, Matagne A. Single-domain antibody fragments with high conformational stability. Protein Sci. 2002;11:500-15.

32. Bannas P, Hambach J, Koch-Nolte F. Nanobodies and Nanobody-Based Human Heavy Chain Antibodies As Antitumor Therapeutics. Front Immunol. 2017;8:1603.

33. Stocki P, Szary J, Rasmussen CLM, Demydchuk M, Northall L, Logan DB, Gauhar A, Thei L, Moos T, Walsh FS, Rutkowski JL. Blood-brain barrier transport using a high affinity, brain-selective VNAR antibody targeting transferrin receptor 1. Faseb j. 2021;35:e21172.

34. Kijanka M, Dorresteijn B, Oliveira S, van Bergen en Henegouwen PM. Nanobody-based cancer therapy of solid tumors. Nanomedicine (Lond). 2015;10:161-74. 
35. Scully M, Cataland SR, Peyvandi F, Coppo P, Knöbl P, Kremer Hovinga JA, Metjian A, de la Rubia J, Pavenski K, Callewaert F, Biswas D, De Winter H, Zeldin RK. Caplacizumab Treatment for Acquired Thrombotic Thrombocytopenic Purpura. N Engl J Med. 2019;380:335-46.

36. Kim YJ, Yoon DS, Jung UJ. Efficacy of nobiletin in improving hypercholesterolemia and nonalcoholic fatty liver disease in high-cholesterol diet-fed mice. Nutr Res Pract. 2021;15:431-43.

37. Sun G, Jackson CV, Zimmerman K, Zhang LK, Finnearty CM, Sandusky GE, Zhang G, Peterson RG, Wang YJ. The FATZO mouse, a next generation model of type 2 diabetes, develops NAFLD and NASH when fed a Western diet supplemented with fructose. BMC Gastroenterol. 2019;19:41.

38. The diagnosis and management. of nonalcoholic fatty liver disease: Practice guidance from the American Association for the Study of Liver Diseases. Clin Liver Dis (Hoboken). 2018;11:81.

39. Anstee QM, Reeves HL, Kotsiliti E, Govaere O, Heikenwalder M. From NASH to HCC: current concepts and future challenges. Nat Rev Gastroenterol Hepatol. 2019;16:411-28.

40. Francque S, Szabo G, Abdelmalek MF, Byrne CD, Cusi K, Dufour JF, Roden M, Sacks F, Tacke F. Nonalcoholic steatohepatitis: the role of peroxisome proliferator-activated receptors. Nat Rev Gastroenterol Hepatol. 2021;18:24-39.

41. Su X, Cheng Y, Chang D. Lipid-lowering therapy: Guidelines to precision medicine. Clin Chim Acta. 2021;514:66-73.

42. Jin M, Meng F, Yang W, Liang L, Wang H, Fu Z. Efficacy and Safety of Evinacumab for the Treatment of Hypercholesterolemia: A Meta-Analysis. J Cardiovasc Pharmacol. 2021;78:394-402.

43. Musunuru K, Pirruccello JP, Do R, Peloso GM, Guiducci C, Sougnez C, Garimella KV, Fisher S, Abreu J, Barry AJ, Fennell T, Banks E, Ambrogio L, Cibulskis K, Kernytsky A, Gonzalez E, Rudzicz N, Engert JC, DePristo MA, Daly MJ, Cohen JC, Hobbs HH, Altshuler D, Schonfeld G, Gabriel SB, Yue P, Kathiresan S. Exome sequencing, ANGPTL3 mutations, and familial combined hypolipidemia. N Engl J Med. 2010;363:2220-7.

44. Elverdi T, Eskazan AE. Caplacizumab as an emerging treatment option for acquired thrombotic thrombocytopenic purpura. Drug Des Devel Ther. 2019;13:1251-8.

45. Peyvandi F, Scully M, Kremer Hovinga JA, Cataland S, Knöbl P, Wu H, Artoni A, Westwood JP, Mansouri Taleghani M, Jilma B, Callewaert F, Ulrichts H. Duby C and Tersago D. Caplacizumab for Acquired Thrombotic Thrombocytopenic Purpura. N Engl J Med. 2016;374:511-22.

46. van Faassen H, Ryan S, Henry KA, Raphael S, Yang Q, Rossotti MA, Brunette E, Jiang S, Haqqani AS, Sulea T, MacKenzie CR, Tanha J, Hussack G. Serum albumin-binding $V(H) H$ s with variable $\mathrm{pH}$ sensitivities enable tailored half-life extension of biologics. Faseb j. 2020;34:8155-71.

47. Schneeweis LA, Obenauer-Kutner L, Kaur P, Yamniuk AP, Tamura J, Jaffe N, O'Mara BW, Lindsay S, Doyle M, Bryson J. Comparison of Ensemble and Single Molecule Methods for Particle Characterization and Binding Analysis of a PEGylated Single-Domain Antibody. J Pharm Sci. 2015;104:4015-24.

48. Li X, Wang M, Zhang X, Liu C, Xiang H, Huang M, Ma Y, Gao X, Jiang L, Liu X, Li B, Hou Y, Zhang X, Yang S, Yang N. The novel llama-human chimeric antibody has potent effect in lowering LDL-c levels 
in hPCSK9 transgenic rats. Clin Transl Med. 2020;9:16.

49. Coppieters K, Dreier T, Silence K, de Haard H, Lauwereys M, Casteels P, Beirnaert E, Jonckheere H, Van de Wiele C, Staelens L, Hostens J, Revets H, Remaut E, Elewaut D, Rottiers P. Formatted anti-tumor necrosis factor alpha $\mathrm{VHH}$ proteins derived from camelids show superior potency and targeting to inflamed joints in a murine model of collagen-induced arthritis. Arthritis Rheum. 2006;54:1856-66.

50. Wrapp D, De Vlieger D, Corbett KS, Torres GM, Wang N, Van Breedam W, Roose K, van Schie L, Team V-CC-R, Hoffmann M, Pohlmann S, Graham BS, Callewaert N, Schepens B. Saelens X and McLellan JS. Structural Basis for Potent Neutralization of Betacoronaviruses by Single-Domain Camelid Antibodies. Cell. 2020;181:1004-15 e15.

51. Tu LN, Showalter MR, Cajka T, Fan S, Pillai VV, Fiehn O, Selvaraj V. Metabolomic characteristics of cholesterol-induced non-obese nonalcoholic fatty liver disease in mice. Sci Rep. 2017;7:6120.

52. Usui S, Hara Y, Hosaki S, Okazaki M. A new on-line dual enzymatic method for simultaneous quantification of cholesterol and triglycerides in lipoproteins by HPLC. J Lipid Res. 2002;43:805-14.

\section{Figures}

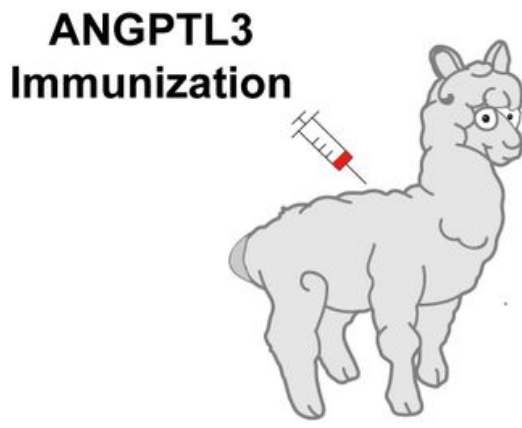

ANGPTL3

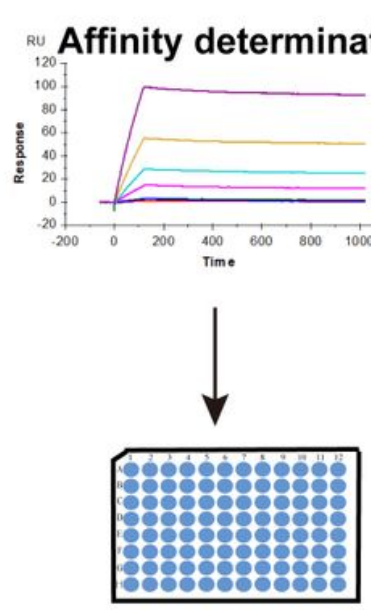

In vitro LPL assay

\section{on}

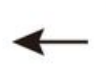

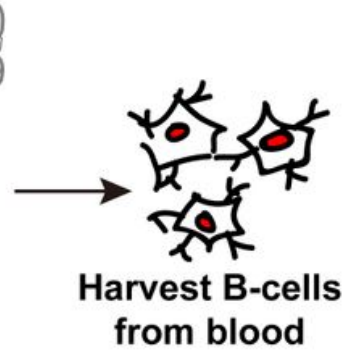

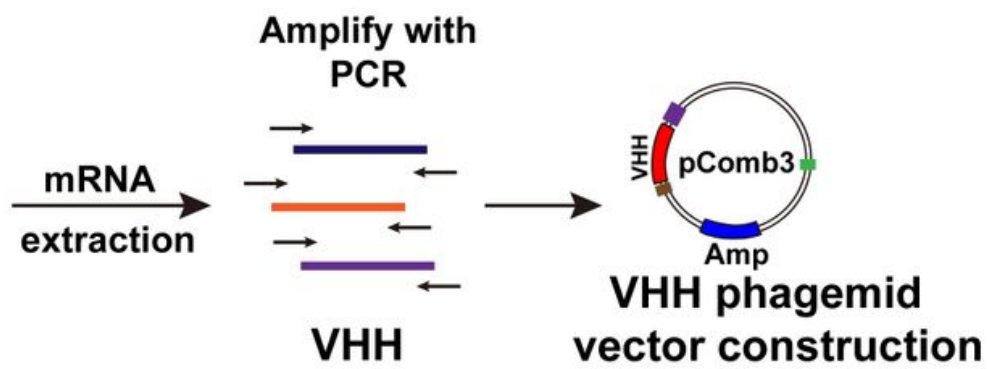

VHH

vector construction

TG1 E.coli

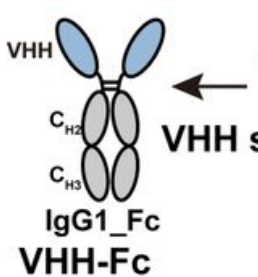

WOOW $\leftarrow$
sanger sequening
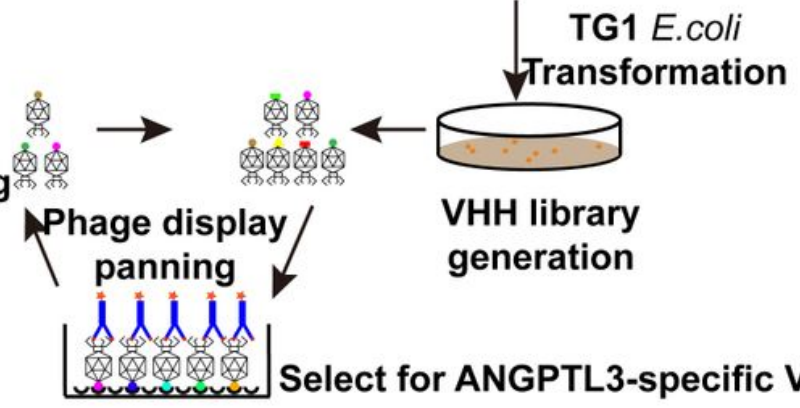

Transformation

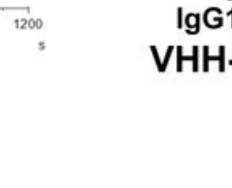

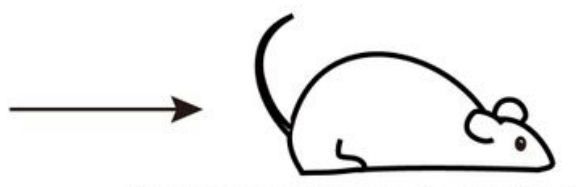

Pharmacodynamic evaluation in Hypercholesterolemia

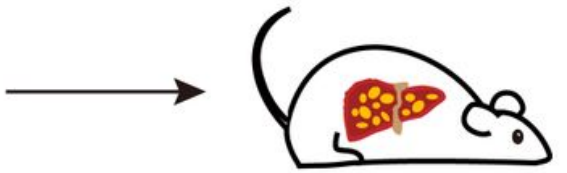

Pharmacodynamic evaluation in NAFLD

Figure 1

Schematic presentation of the whole experiment. The whole experimental procedure includes ANGPTL3 immunization, harvest B-cells separation, VHH library generation, phage-display to select ANGPTL3- 
A
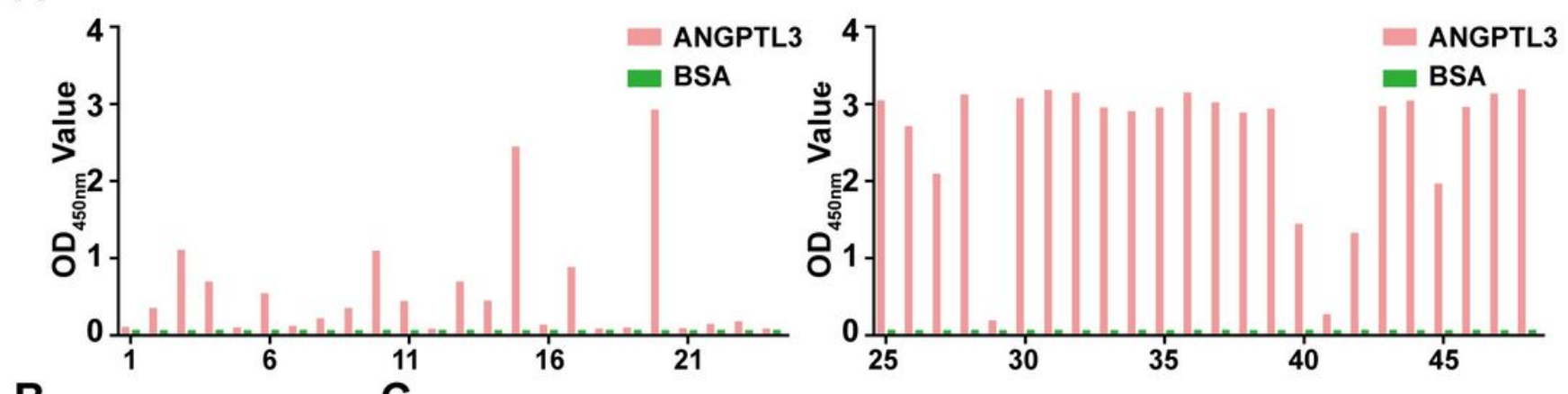

B

C

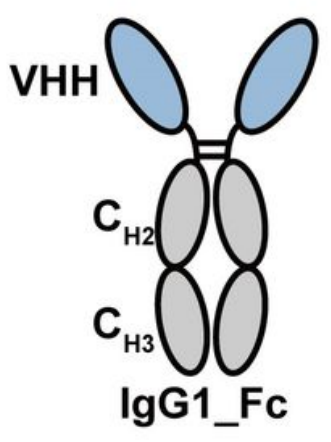

kDaM $1 \begin{array}{lllllllll} & 2 & 3 & 4 & \mathrm{kDaM} & 5 & 6\end{array}$

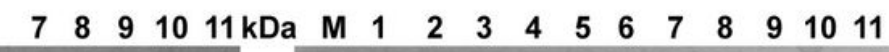

D

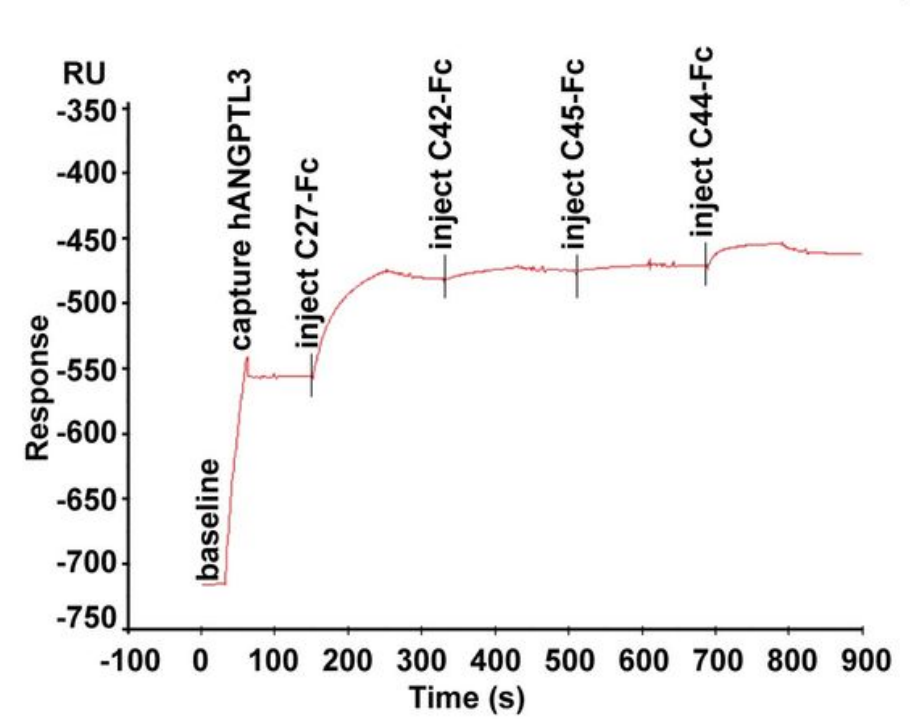

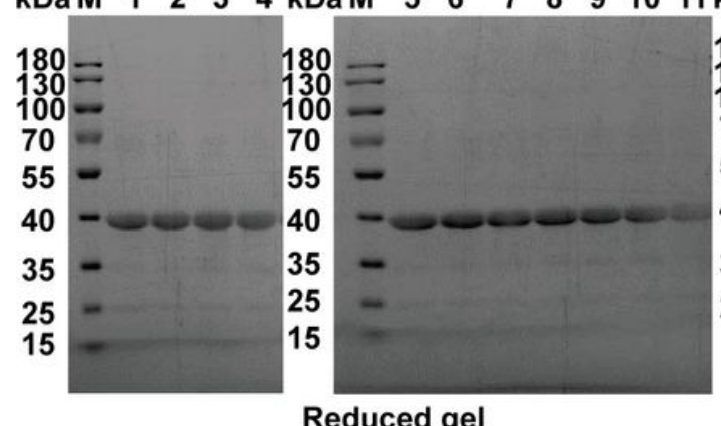

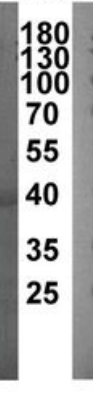

$180=$
$130=$
$100=$
70
55
$40=$
$35=$
25

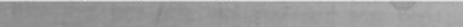

$\mathbf{E}$

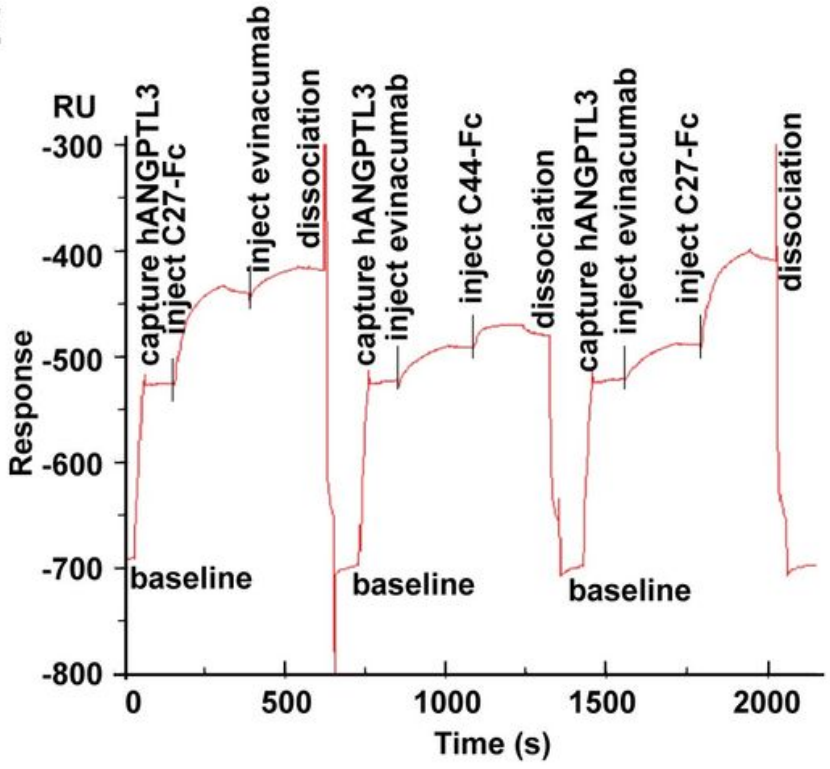

Figure 2

Screening and binding epitopes test of the anti-hANGPTL3 (S17-K170)-mFc VHH-Fc chimeric antibodies. (A) Identification of ANGPTL3 specific VHHs from 48 clones specifically binding with hANGPTL3 after three rounds bio-panning. (B) Diagram of the VHHs fused with human IgG1 Fc fragments. (C) The SDSPAGE analysis of the C3-Fc, C10-Fc, C25-Fc, C26-Fc, C27-Fc, C28-Fc, C30-Fc, C42-Fc, C44-Fc, C45-Fc, C46Fc.M: Maker; lane 1: C3-Fc; lane 2: C10-Fc; lane 3: C25-Fc; lane 4: C26-Fc; lane 5: C27-Fc; lane 6: C28-Fc; lane 7: C30-Fc; lane 8: C42-Fc; lane 9: C44-Fc; lane 10: C45-Fc; lane 11: C46-Fc. (D) C27-Fc, C42-Fc, C44-Fc 
and C45-Fc were tested by SPR for the hANGPTL3-binding epitopes assay. (E) C27-Fc and C44-Fc were tested against evinacumab by SPR for the hANGPTL3-binding epitopes assay. The vertical and horizontal coordinate axis respectively indicate the response unit and timeline. The curves represent procedures of baseline, hANGPTL3 (S17-K170) captured, antibodies injected or dissociation. hANGPTL3 represent human ANGPTL3.

A

C27-Fc binding to hANGPTL3 (S17-K170)

$B_{\text {RU }}$ C27-Fc binding to hANGPTL3 (S17-E460)

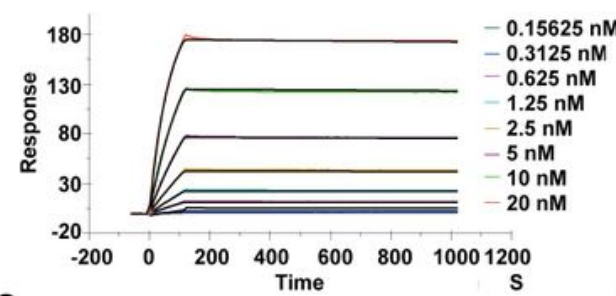

C RU C27-Fc binding to mANGPTL3 (S17-T206)
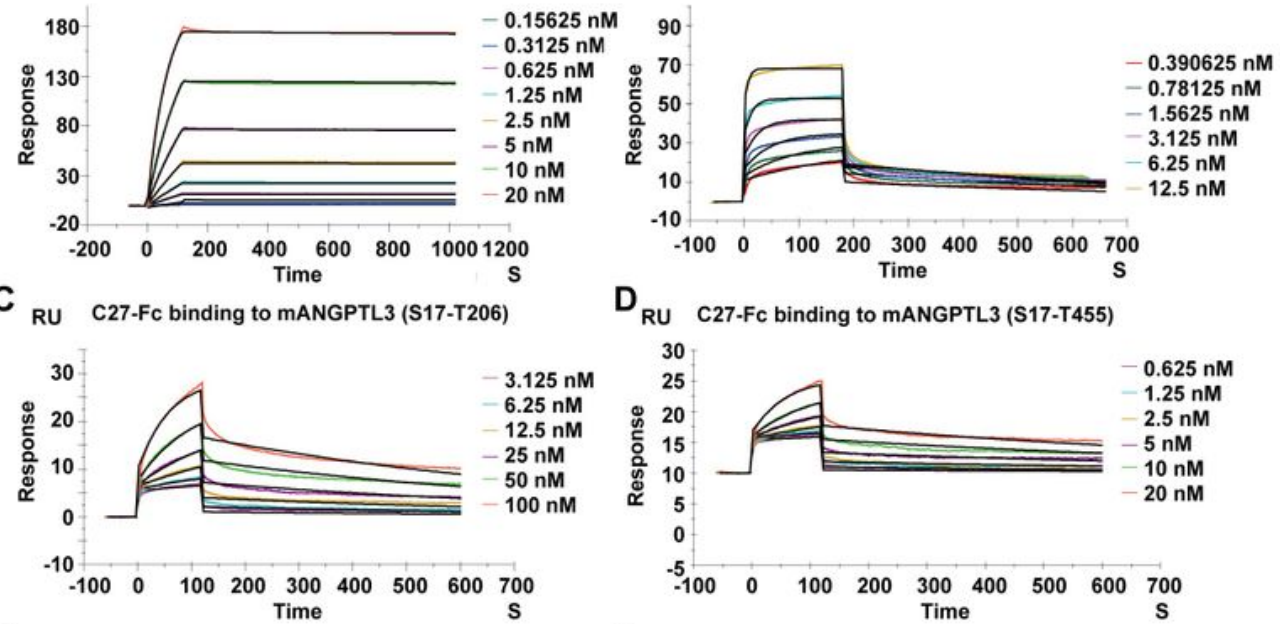

E

D $_{\text {RU C27-Fc binding to mANGPTL3 (S17-T455) }}$

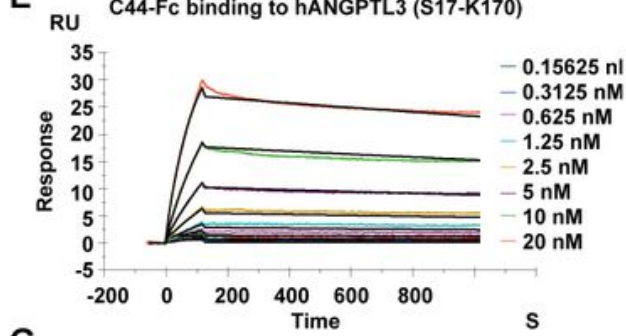

G

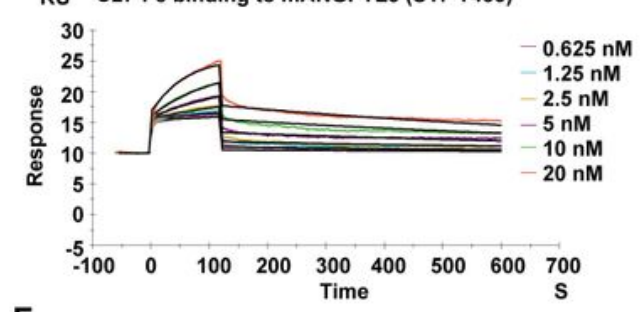

$F_{\text {RU C44-Fc binding to hANGPTL3 (S17-E460) }}$

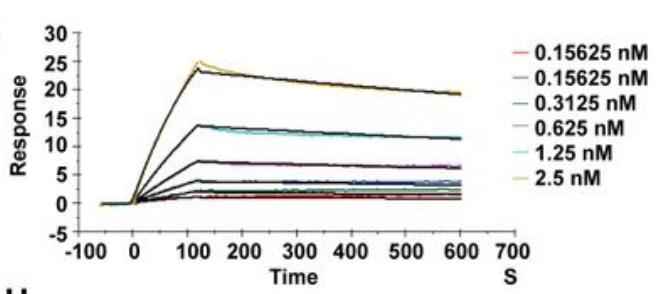

$\mathrm{H}_{\text {RU C44-Fc binding to mANGPTL3 (S17-T455) }}$

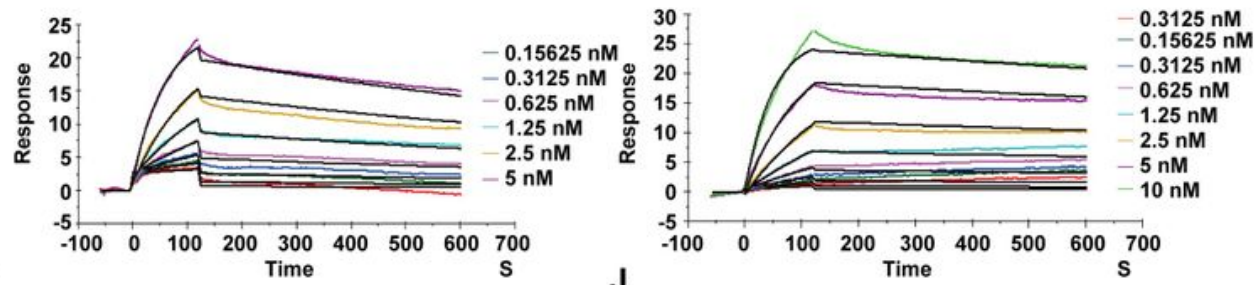

RU Evinacumab binding to hANGPTL3 (S17-K170)

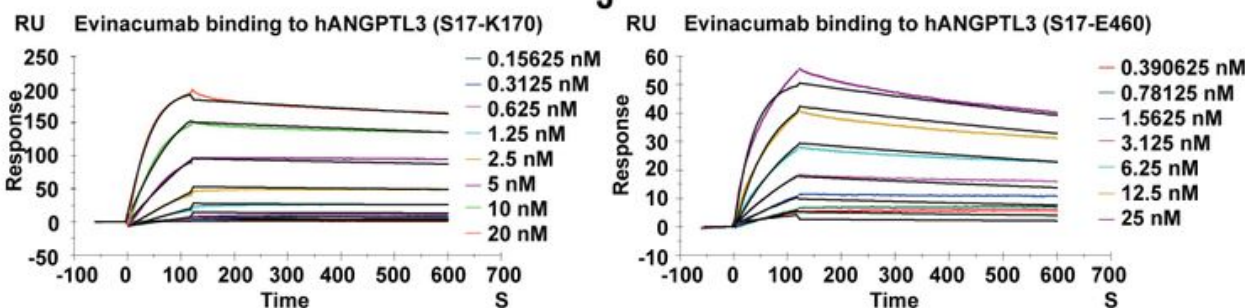

K RU Evinacumab binding to mANGPTL3 (S17-T206) $L_{\text {RU }}$ Evinacumab binding to mANGPTL3 (S17-T455)

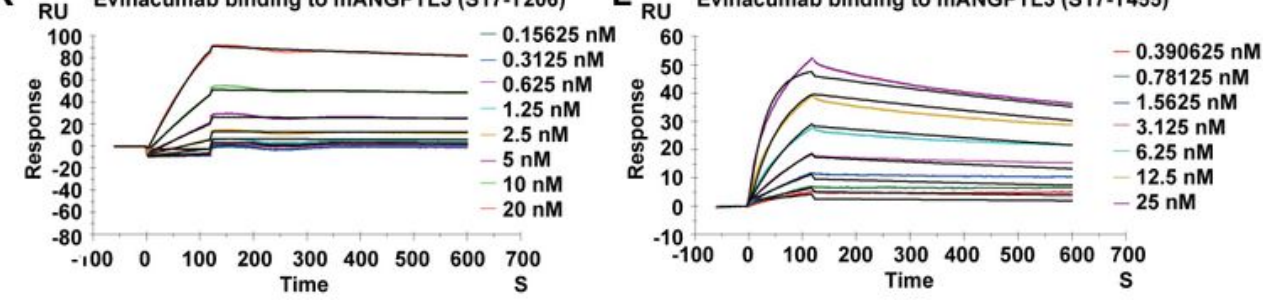

Figure 3 
The affinity of C27-Fc and C44-Fc with human and mouse ANGPTL3 proteins. (A, E and I) The affinity of C27-Fc, C44-Fc and evinacumab binding to hANGPTL3 (S17-K170). The lines with different colors show different concentration of hANGPTL3 (S17-K170) diluted by double fold. (B, F and J) The affinity of C27Fc, C44-Fc and evinacumab binding to hANGPTL3 (S17-E460). (C, G and K) The affinity of C27-Fc, C44-Fc and evinacumab binding to mANGPTL3 (S17-T206). (D, H and L) The affinity of C27-Fc, C44-Fc and evinacumab binding to mANGPTL3 (S17-T455). The lines with different colors show different concentration of VHHs-Fc and evinacumab diluted by double fold. mANGPTL3 represents mouse ANGPTL3.

\section{Figure 4}

The biophysical stability evaluation of C27-Fc and C44-Fc in vitro. (A) SEC-HPLC profile of C27-Fc, C44-Fc and evinacumab (B) Thermal stability analysis of C27-Fc, C44-Fc and evinacumab (C) The expression levels of $\mathrm{C} 44-\mathrm{Fc}$, evinacumab and control $\mathrm{Ab}$ in ExpiCHO cells. The supernatants of ExpiCHO cells instantaneously transfected with $\mathrm{C} 44-\mathrm{Fc}$, evinacumab and control $\mathrm{Ab}$ expression plasmid were collected on $5,7,12$ days. (D and E) SEC-HPLC analysis of C44-Fc and evinacumab at low or high pH. C44-Fc and evinacumab were preserved at $\mathrm{pH} 5.0$ or $\mathrm{pH} 9.0$ and $40{ }^{\circ} \mathrm{C}$ for $72 \mathrm{~h}$. (F) SEC-HPLC analysis of C44-Fc and evinacumab at $40{ }^{\circ} \mathrm{C}$ for 28 days. (G) The freeze-thaw stability analysis of C44-Fc and evinacumab by SEC-HPLC.
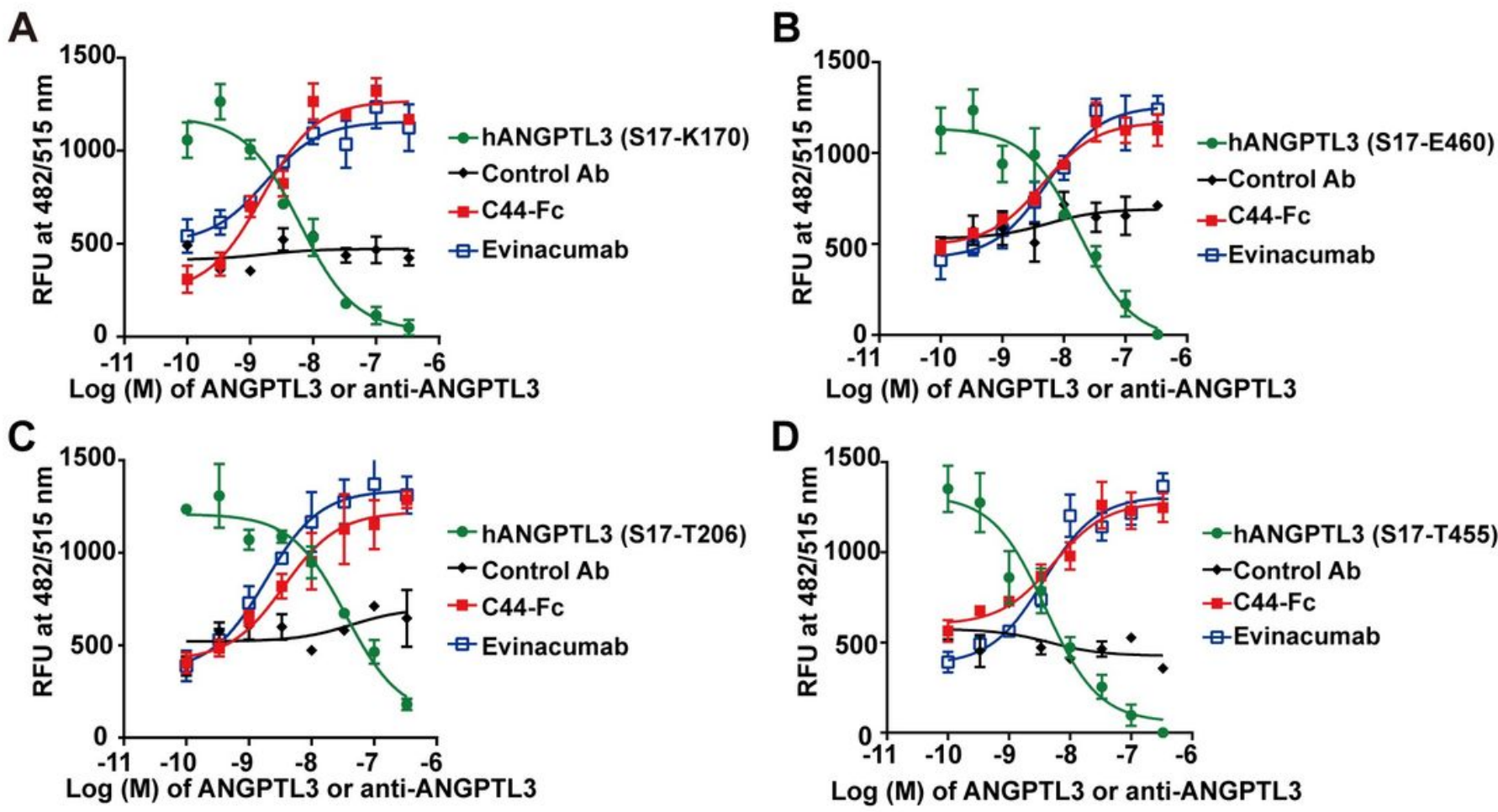

Figure 5 
C44-Fc suppresses ANGPTL3-induced blockade of LPL. The vertical axis indicates the incremental concentrations of four kinds of ANGPTL3 proteins or antibody including of C44-Fc, evinacumab and control $A b(n=3)$. The vertical axis indicates the relative fluorescence units (RFU) at 482/515 nm (excitation/emission).

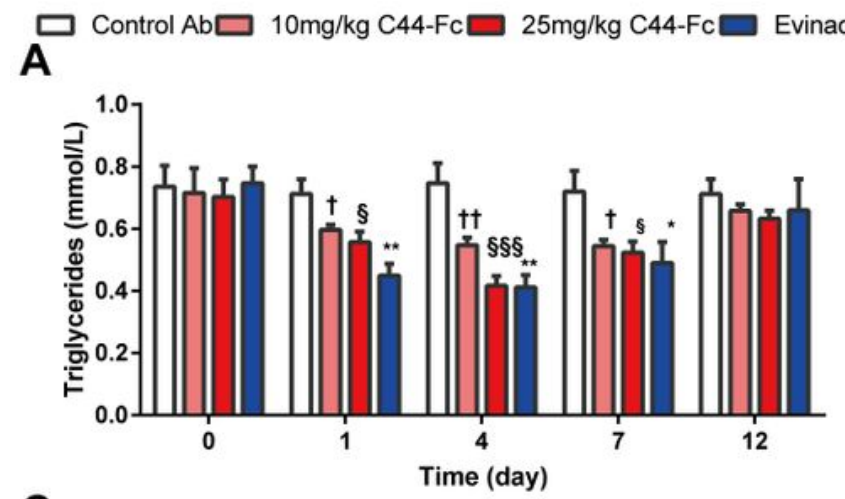

C

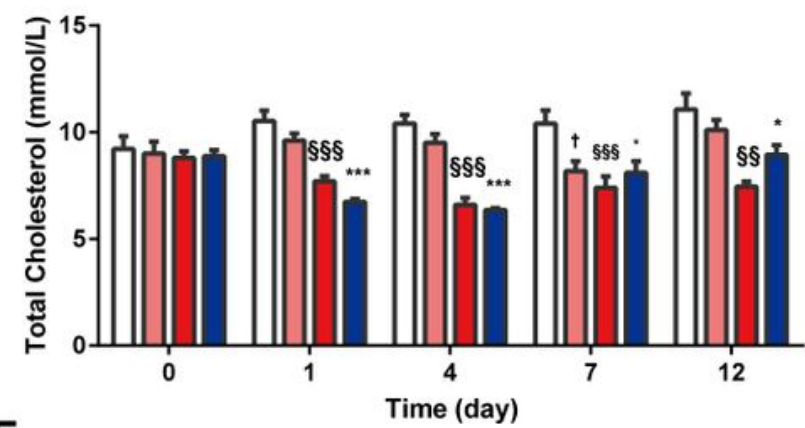

$\mathbf{E}$

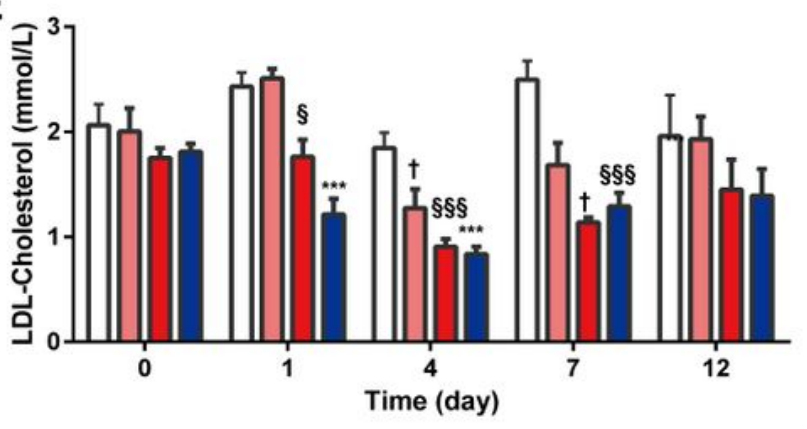

B

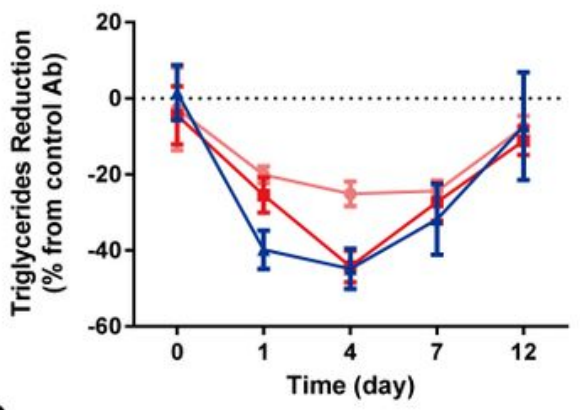

D
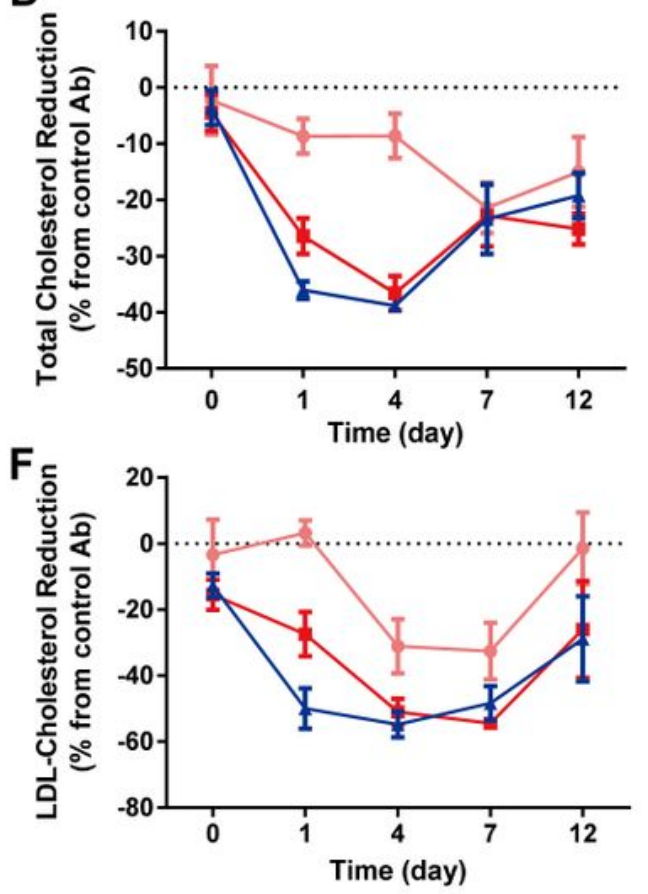

G

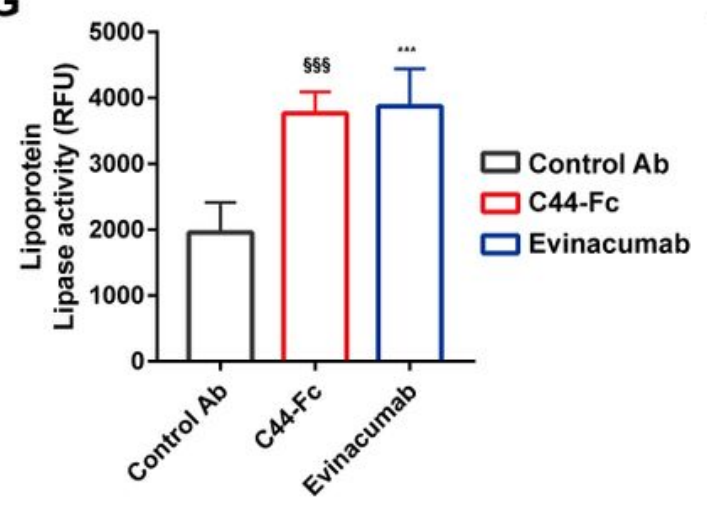

H

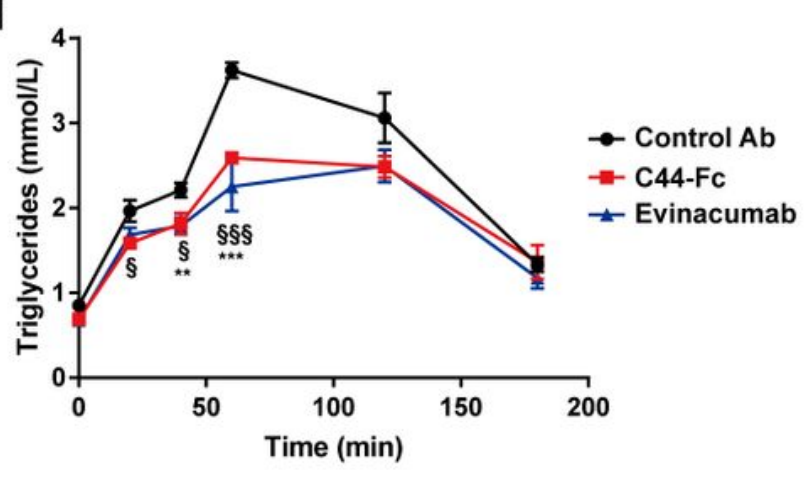

Figure 6 
C44-Fc reduces serum TG, TC and LDL-C levels in hypercholesterolemic mice. C57BL/6 mice were induced by HF/HCCD-fed for four weeks to establish hypercholesterolemic mice model and then treated with $25 \mathrm{mg} / \mathrm{kg}$ isotype control Ab, $10 \mathrm{mg} / \mathrm{kg}$ C44-Fc, $25 \mathrm{mg} / \mathrm{kg}$ C44-Fc, $25 \mathrm{mg} / \mathrm{kg}$ evinacumab. Serum TG $(A), T C(C)$ and LDL-C (E) levels were tested enzymatically $(n=6-8)$. The TG (B), TC (D) and LDL-C (F) reduction levels of $\mathrm{C} 44-\mathrm{Fc}$ and evinacumab from control $\mathrm{Ab}$ were also measured. (G) The plasma $\mathrm{LPL}$ activities after heparin intravenous injection in tail $(n=6)$. $(H)$ Lipid tolerance test were performed four days after $25 \mathrm{mg} / \mathrm{kg}$ of control Ab, C44-Fc and evinacumab treatment $(n=6) .+$, $\S$ and * respectively represent the Student's t-test of $10 \mathrm{mg} / \mathrm{kg} \mathrm{C} 44-\mathrm{Fc}, 25 \mathrm{mg} / \mathrm{kg}$ C44-Fc and $25 \mathrm{mg} / \mathrm{kg}$ evinacumab treatment compared with control Ab. 
Drug injection:once a week; Physical examination;

Serum assay.

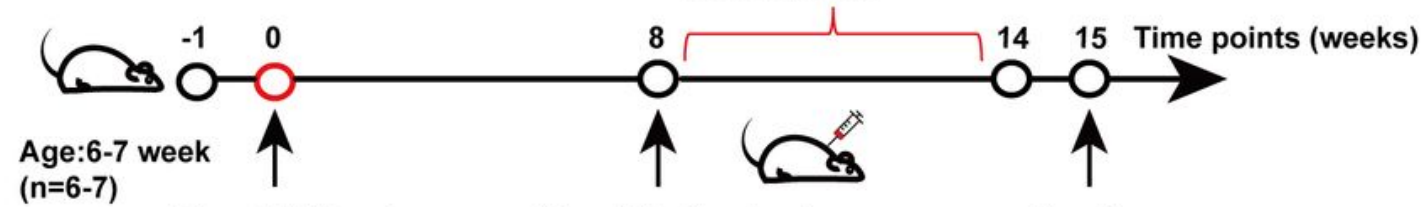

Chow/HFD begin

Drug injection begin

Control Ab

C44-Fc

Evinacumab
Sacrifice;

Sample collection.

$\rightarrow$ Chow $\quad$ Control Ab $\quad-$ C44-Fc $\rightarrow$ Evinacumab

B

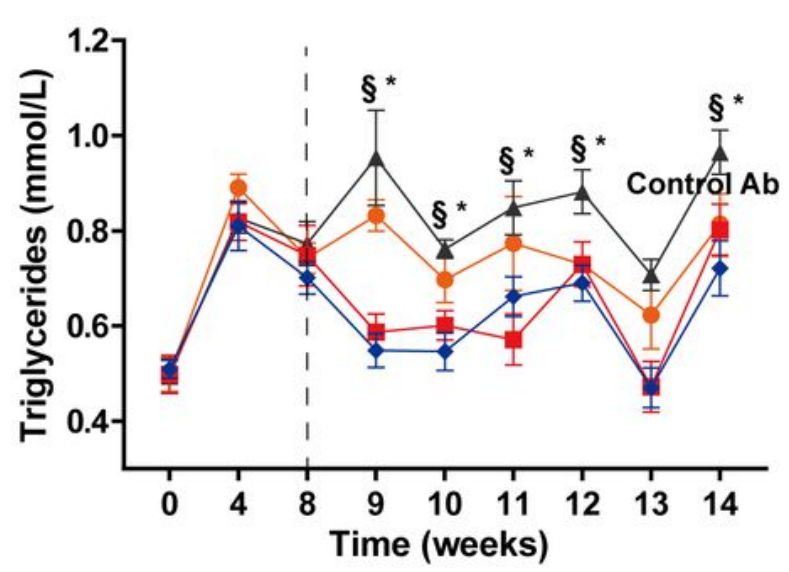

D

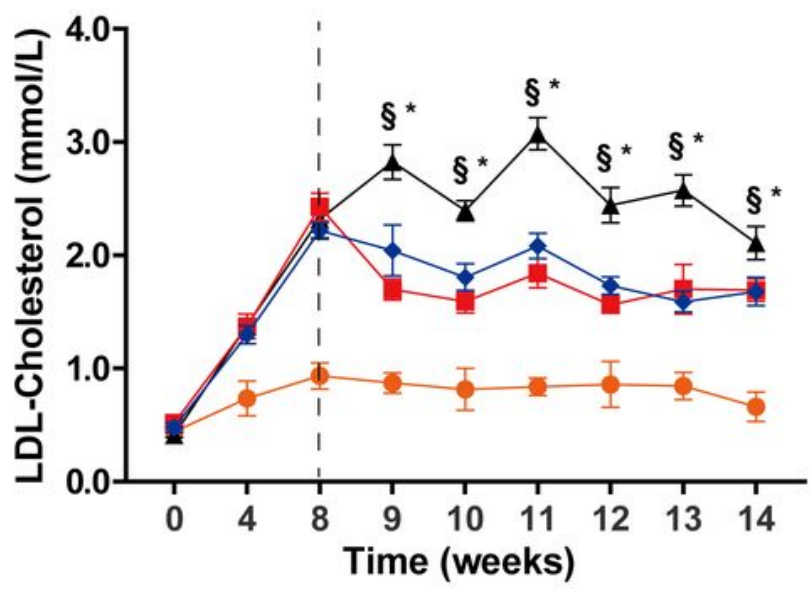

C

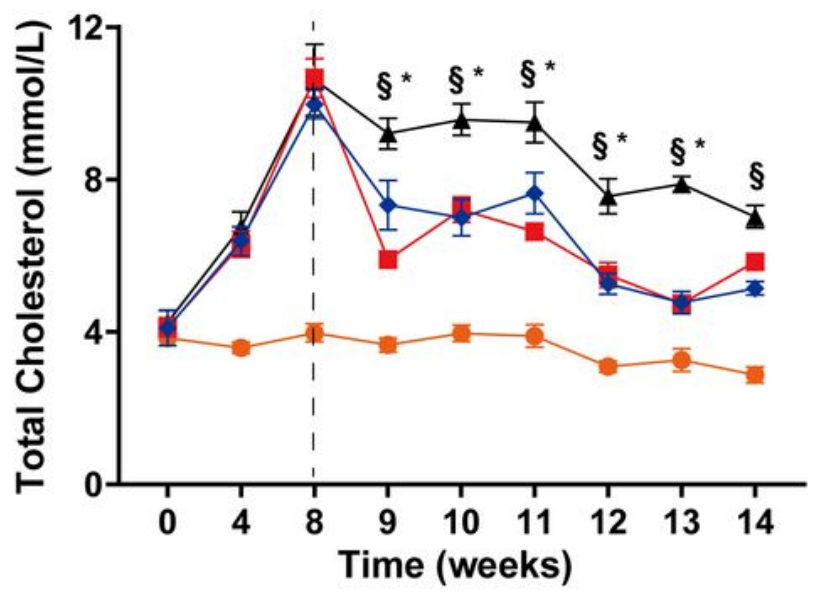

E

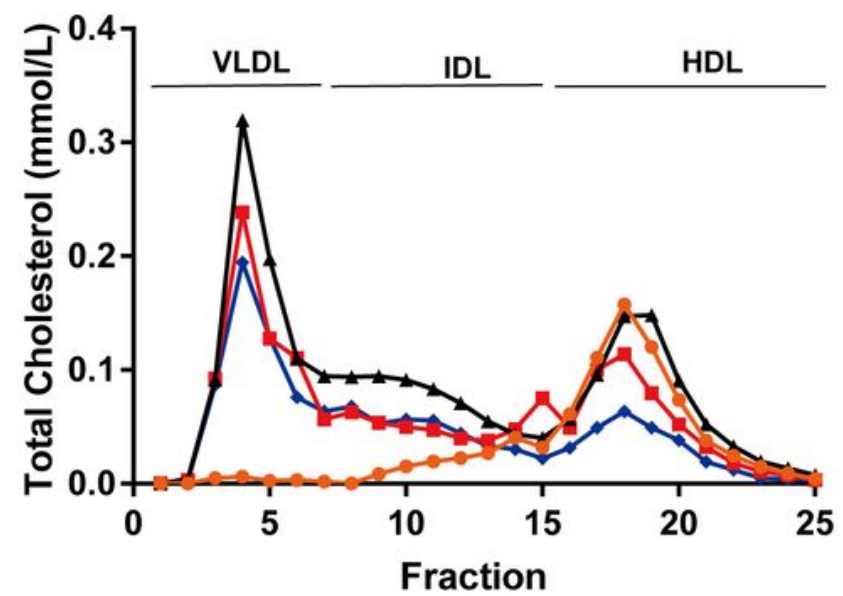

Figure 7

C44-Fc administrations reduce serum lipid levels for six weeks. (A) Schematic illustration of experimental design to assess the treatment of C44-Fc multiple administrations. C57BL/ 6 mice were induced by a $\mathrm{HF} / \mathrm{HCCD}$-fed for eight weeks and then treated with $25 \mathrm{mg} / \mathrm{kg}$ of isotype control Ab, $25 \mathrm{mg} / \mathrm{kg}$ of C44-Fc, $25 \mathrm{mg} / \mathrm{kg}$ of evinacumab weekly. Serum TG (B), TC (C) and LDL-C (D) levels were weekly tested four days after injections $(n=6)$. Plasma lipoproteins of cholesterol (E) Plasma lipoproteins of TC were separated 
by HPLC. $\S$ and * respectively represent the Student's t-test of $25 \mathrm{mg} / \mathrm{kg} \mathrm{C44-Fc} \mathrm{and} 25 \mathrm{mg} / \mathrm{kg}$ evinacumab treatment compared with control Ab.

A

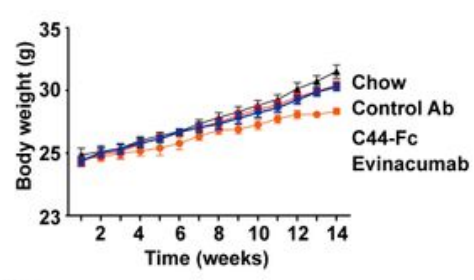

C

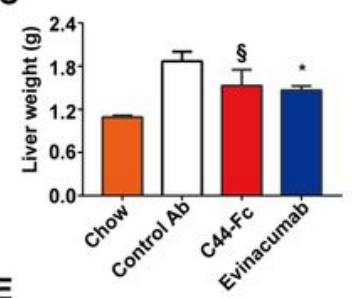

E

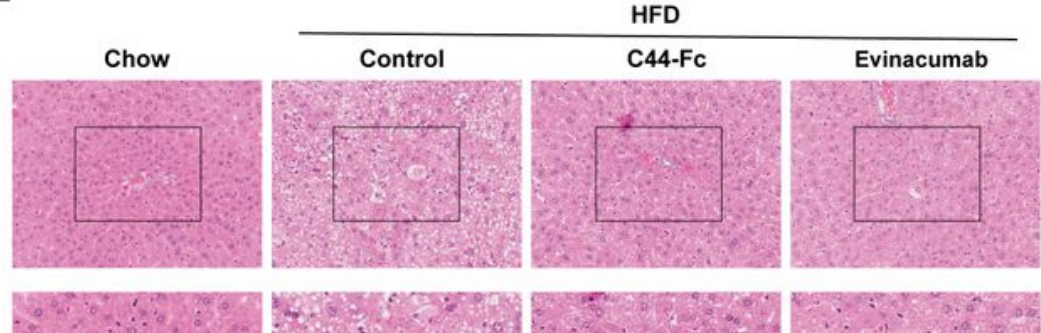

B

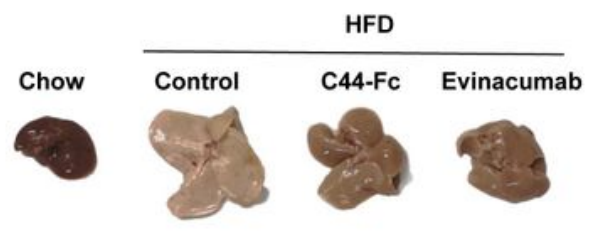

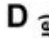

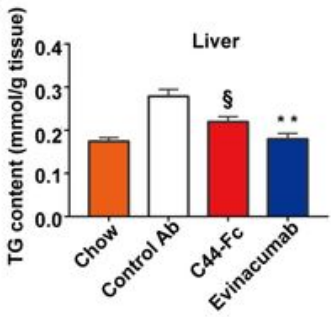


livers were measured $(n=5)$ by commercial kits. (E) Representative H\&E staining of liver tissues. $(F)$ Representative images and $(G)$ quantification $(n=3)$ of oil red $O$ staining are presented. The levels of Serum ALT $(H)$ and AST (I) were measured at the terminus of the research $(n=6)$. $\S$ and * respectively represent the Student's t-test of $25 \mathrm{mg} / \mathrm{kg}$ C44-Fc and $25 \mathrm{mg} / \mathrm{kg}$ evinacumab treatment compared with control Ab.

\section{Supplementary Files}

This is a list of supplementary files associated with this preprint. Click to download.

- renamed2161c.jpg

- Additionalfile1.docx 